\title{
Ultramassive dense early-type galaxies: Velocity dispersions and number density evolution since $z=1.6$
}

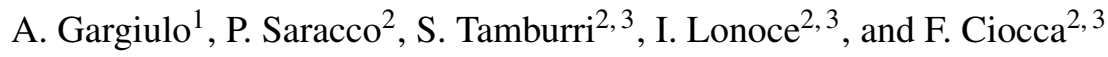 \\ 1 INAF-Istituto di Astrofisica Spaziale e Fisica Cosmica Milano, via Bassini 15, 20133 Milano, Italy \\ e-mail: adriana@lambrate.inaf.it \\ 2 INAF-Osservatorio Astronomico di Brera, via Brera 28, 20121 Milano, Italy \\ 3 Dipartimento di Scienza e Alta Tecnologia, Università degli Studi dell'Insubria, via Valleggio 11, 22100 Como, Italy
}

Received 20 May 2015 / Accepted 3 May 2016

\begin{abstract}
Aims. We investigate the stellar mass assembly history of ultramassive $\left(M_{\star} \gtrsim 10^{11} M_{\odot}\right)$ dense $\left(\Sigma=M_{\star} / 2 \pi R_{\mathrm{e}}^{2}>2500 M_{\odot} \mathrm{pc}^{-2}\right)$ early-type galaxies (ETGs, elliptical and spheroidal galaxies) selected on basis of visual classification over the last $9 \mathrm{Gyr}$.

Methods. We traced the evolution of the comoving number density $\rho$ of ultramassive dense ETGs and compared their structural (effective radius $R_{\mathrm{e}}$ and stellar mass $M_{\star}$ ) and dynamical (velocity dispersion $\sigma_{\mathrm{e}}$ ) parameters over the redshift range $0<z<1.6$. We derived the number density $\rho$ at $1.6<z<1$ from the MUNICS and GOODS-South surveys, while we took advantage of the COSMOS spectroscopic survey to probe the intermediate redshift range [0.2-1.0]. We derived the number density of ultramassive dense local ETGs from the SDSS sample taking all of the selection bias affecting the spectroscopic sample into account. To compare the dynamical and structural parameters, we collected a sample of 11 ultramassive dense ETGs at $1.2<z<1.6$ for which velocity dispersion measurements are available. For four of these ETGs (plus one at $z=1.91$ ), we present previously unpublished estimates of velocity dispersion, based on optical VLT-FORS2 spectra. We probe the intermediate redshift range $(0.2 \lessgtr z \lessgtr 0.9)$ and the local Universe with different ETGs samples.

Results. We find that the comoving number density of ultramassive dense ETGs evolves with $z$ as $\rho(z) \propto(1+z)^{0.3 \pm 0.8}$ implying a decrease of $\sim 25 \%$ of the population of ultramassive dense ETGs since $z=1.6$. By comparing the structural and dynamical properties of high- $z$ ultramassive dense ETGs over the range $0 \lesssim z<1.6$ in the $\left[R_{\mathrm{e}}, M_{\star}, \sigma_{\mathrm{e}}\right]$ plane, we find that all of the ETGs of the high- $z$ sample have counterparts with similar properties in the local Universe. This implies either that the majority $(\sim 70 \%)$ of ultramassive dense ETGs already completed the assembly and shaping at $\langle z\rangle=1.4$, or that, if a significant portion of dense ETGs evolves in size, new ultramassive dense ETGs must form at $z<1.5$ to maintain their number density at almost constant. The difficulty in identify good progenitors for these new dense ETGs at $z \lesssim 1.5$ and the stellar populations properties of local ultramassive dense ETGs point towards the first hypothesis. In this case, the ultramassive dense galaxies missing in the local Universe could have joined, in the last 9 Gyr, the so colled non-dense ETGs population through minor mergers, thus contributing to mean size growth. In any case, the comparison between their number density and the number density of the whole population of ultramassive ETGs relegates their contribution to the mean size evolution to a secondary process.
\end{abstract}

Key words. galaxies: elliptical and lenticular, cD - galaxies: evolution - galaxies: formation - galaxies: high-redshift

\section{Introduction}

Most of the studies focusing on the structural parameters of massive, passive galaxies, both at high and at low redshift, indicate that this population of galaxies has increased the mean effective radius over the cosmic time (e.g. Daddi et al. 2005; Trujillo et al. 2006, 2007; Longhetti et al. 2007; Zirm et al. 2007; Toft et al. 2007; van Dokkum et al. 2008; Franx et al. 2008; Cimatti et al. 2008; Buitrago et al. 2008; van der Wel et al. 2008, 2014; Bernardi 2009; Damjanov et al. 2009; Bezanson et al. 2009; Mancini et al. 2009, 2010; Cassata et al. 2011; Damjanov et al. 2011; Bruce et al. 2012; Szomoru et al. 2012; Newman et al. 2012; Cassata et al. 2013; Belli et al. 2014). For example, van der Wel et al. (2014) find that the mean effective radius $\left\langle R_{\mathrm{e}}\right\rangle$ of passive galaxies selected with a colour-colour criterion increases as $\left\langle R_{\mathrm{e}}\right\rangle \propto(1+z)^{-1.48}$ over the redshift range $0<z<3$. Similar results, among the others, have also been reached by Cassata et al. (2013) on samples of passive (specific star formation rate $S S F R<10^{-11} \mathrm{yr}^{-1}$ ) galaxies, and by Cimatti et al. (2012) for galaxies with red colours and elliptical morphology. Nonetheless, other analyses contrast these results, showing a mild or an even negligible evolution of the mean size with cosmic time (Saracco et al. 2011, 2014; Stott et al. 2011; Jorgensen \& Chiboucas 2013; Jorgensen et al. 2014; Poggianti et al. 2013a). Most of these works investigate a sample of galaxies selected on the basis of their elliptical morphology.

In fact, the reason for these different results is not easily detectable. A major point to be considered is that a selection based on the passivity (or on the Sersic index $n>2.5$; see e.g. Buitrago et al. 2013) includes in the sample a significant portion $(>30 \%)$ of disk galaxies (e.g. van der Wel et al. 2011; Ilbert et al. 2010; Cassata et al. 2011; Tamburri et al. 2014) and, concurrently, does not include all of the galaxies that are visually classified as ellipticals. At the same time, a selection based on a cut in SSFR that is constant with time (usually $S S F R<$ $10^{-11} \mathrm{yr}^{-1}$ ) selects predominantly red spheroids at $z \sim 2$, but includes a large contamination of disk/spiral galaxies in the local Universe (Szomoru et al. 2011). Actually, Keating et al. (2015) 
show that the properties of high- $z$ elliptical galaxies are highly sensitive to the definitions used in selecting the samples (see also Moresco et al. 2013).

In addition to this debated issue on the amount of evolution in size, its possible origin is also investigated. Is the increase of the mean size due to the growth in size of individual galaxies across the time, resulting from inside-out stellar matter accretion due to minor dry merging, or rather to the addition of new and larger galaxies over cosmic time (e.g. Valentinuzzi et al. 2010; Carollo et al. 2013)? A promising approach to shed light on the origin of the size evolution is to investigate how the number density of dense galaxies evolves over the cosmic time (Saracco et al. 2010; Bezanson et al. 2012; Cassata et al. 2013; Poggianti et al. 2013b; Carollo et al. 2013; Damjanov et al. 2014, 2015). The analyses by various authors differ slightly in the selection of the sample, for example via passivity, visual classification, colour, and Sersic index; in the definition of so-called dense galaxies, selected for example on the basis of their mean stellar mass density $\Sigma=M_{\star} / 2 \pi R_{\mathrm{e}}^{2}$ or sigma deviation from the local size-mass relation (SMR); and in the treatment of the progenitor bias. Saracco et al. (2010) find that the number density of dense ( $\sigma$ below the local SMR) massive $\left(M_{\star}>3 \times 10^{10} M_{\odot}\right)$ ellipticals (visually selected) at $0.9<z<1.9$ is comparable with that of equally dense massive ellipticals in local clusters. Similarly, Poggianti et al. (2013b), studying the number density of massive $\left(M_{\star} \gtrsim 10^{10} M_{\odot}\right)$, compact $\left(\log \left(M_{\star} / R_{\mathrm{e}}^{-1.5}\right) \geq 10.3 M_{\odot} \mathrm{kpc}^{-1.5}\right)$, local old galaxies, find that it is just a factor 2 lower than that of compact passive galaxies observed at $z=2$. In contrast, van der Wel et al. (2014) find that the number density of dense $\left(R_{\mathrm{e}} /\left(M_{\star} / 10^{11} M_{\odot}\right)<1.5 \mathrm{kpc}\right)$, massive $\left(M_{\star}>5 \times 10^{10} M_{\odot}\right)$, passive galaxies decreases by a factor $\sim 10$ from $z \sim 2$ at $z \sim 0.2$.

Actually, the individual size growth is expected to be more efficient for the most massive $\left(M_{\star}>10^{11}\right)$ galaxies. In fact, these systems are expected to host the centre of the most massive halo and to evolve mainly through minor mergers (Hopkins et al. 2009). However, given the fact that at each redshift the most massive galaxies are extremely rare (see, e.g., Muzzin et al. 2013), very few works have investigated the number density evolution of their dense subpopulation over cosmic time. Carollo et al. (2013) find that the number density of passive $\left(s S F R<10^{-11} \mathrm{yr}^{-1}\right)$ and elliptical galaxies with $M_{\star}>$ $10^{11} M_{\odot}$ and $R_{\mathrm{e}}<2 \mathrm{kpc}$ decreases $\sim 30 \%$ in the $5 \mathrm{Gyr}$ between $0.2<z<1$. An even mild evolution is claimed by Damjanov et al. (2015), who find an almost constant number density in the range $0.2<z<0.8$ for the passive (colour selected) and dense $\left(\log \left(M_{\star} / R_{\mathrm{e}}^{-1.5}\right) \geq 10.3 M_{\odot} \mathrm{kpc}^{-1.5}\right)$ galaxies with $M_{\star}>8 \times 10^{10} M_{\odot}$.

A study still missing concerns the evolution of the number density of the population of "pure" elliptical (elliptical and spheroidal galaxies selected on the basis of visual classification), massive $\left(M_{\star}>10^{11} M_{\odot}\right)$, dense galaxies, in particular, at $z>0.8-1$, which is the range in which most of the evolution of spheroidal galaxies occurs. From here on, with the term early-type galaxy (ETG) we identify an elliptical galaxy selected on the basis of visual classification, and with no other criterion (e.g. color, Sersic index, sSFR).

In this paper, we aim to probe the mass accretion history of these most massive and dense $\left(M_{\star}>10^{11} M_{\odot}, \Sigma>\right.$ $2500 M_{\odot} \mathrm{pc}^{-2}$ ) ETGs over the previous $\sim 9$ Gyr, by tracing the evolution of their comoving number density and comparing their structural and dynamical parameters from redshift 1.6 to $\sim 0$. One of the aspects of this work that differs from the previous analyses is the selection of the samples based on visual classification, or on a methodology that can mimic the visual classification, over the whole redshift range. In fact, the star formation histories of galaxies are often more complex than we are able to model them, as they are characterized by bursty against smooth and gentle declining star formation histories. As a consequence of this, in a phase of low star formation activity, an active galaxy can just "temporarily" show red colours and/or low sSFR. Moreover, galaxies tend to become passive with time. Hence, the population of passive galaxies collects a different mix of morphological types at different redshift. This leads to a non-homogeneous comparison of their properties at different time (see, e.g., Huertas-Company et al. 2013). In this context, the spheroidal shape of a galaxy is a more stable property over time and allows us to select the same population over cosmic time. In addition, we selected dense galaxies on the basis of their $\Sigma$ rather then using a constant cut in $R_{\mathrm{e}}$ or on the basis of their deviation with respect to the local SMR. The main reason for this choice is that the mean stellar mass density, up to $z \sim 1.5$, is better correlated to, for example the age, the sSFR of the galaxy (Franx et al. 2008; Wake et al. 2012) and hence constitutes a more meaningful parameter.

We organized the analysis into two parts.

First we focus (see Sect. 2) on the evolution of the number density of ultramassive dense ETGs over the redshift range $0<z<1.6$ to investigate whether the ultramassive dense high- $z$ ETGs are numerically consistent with those observed at $z \sim 0$ or, on the contrary, whether they numerically decrease along the time, which would suggest individual growth of galaxies. For this purpose, we have referred to SDSS DR4 survey to constrain the number density of ultramassive dense ETGs at $z \sim 0.1$, paying particular attention to the bias affecting the catalogue (see Sect. 2.3) and to COSMOS field to cover the range $0.2 \lesssim z \lesssim 1$ (see Sect. 2.2). For the highest redshift bin $(1.2 \lesssim z<1.6)$, we have considered two different surveys of similar area $\left(\sim 150 \operatorname{arcmin}^{2}\right)$, the GOODS-South (Giavalisco et al. 2004) and the MUNICS (Drory et al. 2001), to average over the cosmic variance (see Sect. 2.1).

In the second part of the paper, we focus on comparing the structural (i.e. $R_{\mathrm{e}}, M_{\star}$ ) and dynamical (i.e. velocity dispersion $\sigma$ ) parameters of ETGs over the last 9 Gyr (see Sect. 3). This analysis aims to asses whether high- $z$ ultramassive dense ETGs have counterparts at lower $z$ that are characterized by the same structural and dynamical properties or, rather, whether some of these are not present at later epochs. To perform this comparison, we selected data from the literature (see Sect. 3.1). Clearly, the request of available velocity dispersion drastically reduces the statistics of the sample at $1.2<z<1.6$ to 11 ETGs. For four of these ETGs (plus one at $z=1.9$ ), we present new measurements of velocity dispersions (see Sect. 3.1). In Sect. 4 we summarize the work and present our results and conclusions.

Throughout the paper we adopt standard cosmology with $H_{0}=70 \mathrm{~km} \mathrm{~s}^{-1} \mathrm{Mpc}^{-1}, \Omega_{\mathrm{m}}=0.3$ and $\Omega_{\lambda}=0.7$, the stellar masses we report are derived using the Chabrier initial mass function (IMF; Chabrier 2003) and the magnitudes are in the $\mathrm{AB}$ system when not otherwise specified.

\section{The number density of ultramassive dense ETGs}

In this section we constrain the stellar mass accretion history of ultramassive ETGs by investigating the evolution of their number density $\rho$ over the last $\sim 9$ Gyr. We cover the redshift range from 1.6 to 0 with different surveys (e.g. with different magnitude cut and completeness) and in Table 1 we summarize their main characteristics, and the references for the main quantities 
A. Gargiulo et al.: Number density evolution of ultra-massive dense early-type galaxies since $z=1.6$

Table 1. Main characteristics of the surveys used to derived the number density evolution from $z=1.6$ to $z \sim 0$.

\begin{tabular}{|c|c|c|c|c|c|c|}
\hline Name & $\begin{array}{l}\text { Selection } \\
\text { criteria }\end{array}$ & $\begin{array}{l}\text { redshift range } \\
\text { probed }\end{array}$ & $\begin{array}{l}M_{\star}>10^{11} M_{\odot} \\
\text { completeness }\end{array}$ & $M_{\star}$ reference & $R_{\mathrm{e}}$ reference & $R_{\mathrm{e}}$ filter \\
\hline MUNICS & $\begin{array}{l}K<20.4 \& \\
R-K \leq 3.6\end{array}$ & $1.2 \lesssim z<1.6$ & $100 \%$ & Sect. 3.1 & (1) & F160W/HST \\
\hline GOODS-South & $K \mathrm{~s}<22.5$ & $0.65<z<1.6$ & $100 \%$ & (2) & (2) & $F 850 L P / \mathrm{HST}$ \\
\hline COSMOS & $F 814 W<22.5$ & $0.2<z<1.0$ & $\begin{array}{c}100 \% \text { up to } z \sim 0.8 \\
\sim 80 \% \text { at } 0.8<z<1.0\end{array}$ & (3) & (4) & $F 814 W / \mathrm{HST}$ \\
\hline SDSS & $r<16.8$ & $0.063<z<0.1$ & $100 \%$ & MPA-JHU(5) & (6) & $r$ \\
\hline
\end{tabular}

Notes. Column 1: name of the survey; Col. 2: selection criteria, Col. 3: redshift range probed by the survey in our analysis; Col. 4: level of completeness for galaxies with $M_{\star}>10^{11} M_{\odot}$ in the redshift range probed; Col. 5 (6): reference adopted for stellar mass $\left(R_{\mathrm{e}}\right)$ estimates. Stellar masses were derived through the fit of the spectral energy distribution, and effective radius fitting a Sersic profile to the images; Col. 7: filter of the images used to derived the $R_{\mathrm{e}}$.

References. (1) Longhetti et al. (2007); (2) Tamburri et al. (2014); (3) Ilbert et al. (2013); (4) Sargent et al. (2007); (5) http://www. mpa-garching.mpg.de/SDSS/DR7/; (6) Blanton et al. (2005).

involved in this part of analysis (i.e. stellar mass and $R_{\mathrm{e}}$ ). As a reminder, we highlight that this analysis aims to probe the evolution of ETGs, i.e. elliptical and spheroidal galaxies selected on the basis of the visual classification. When this classification is not feasible, we explicitly discussed this in the text.

\subsection{The number density of ultramassive dense ETGS at $1 \lesssim z<1.6$}

For the comoving number density of ultramassive dense ETGs at $z>1$, we referred to two samples of ETGs: one selected in the MUNICS-S2F1 field, and published in Saracco et al. (2005) and Longhetti et al. (2007), and the other selected in the GOODS South-field and described in Tamburri et al. (2014).

MUNICS sample - The MUNICS sample consists of seven ultramassive dense ETGs spectroscopically confirmed at $z \sim 1.5$. These galaxies were observed as part of the spectroscopic follow-up of the complete sample of 19 extremely red objects (EROs; $K \lesssim 20.4, R-K \lesssim 3.6$ ) in the S2F1 field (Saracco et al. 2005). We checked whether the cuts both in magnitude and colour of the MUNICS sample affect our selection of ultramassive dense ETGs. Using the sample of ETGs in the GOODSSouth field presented in Tamburri et al. (2014; see below), we verified that a sample of ETGs that is brighter than $K=20.4$ is $100 \%$ complete up to $z \sim 1.6$. The colour cut tends to deprive the massive sample of the less dense systems (in which we are not interested), since the redder the colour of a galaxy, the higher its $\Sigma$ (Franx et al. 2008; Saracco et al. 2011). All of the galaxies of the MUNICS sample, indeed, have $\Sigma>2500 M_{\odot} \mathrm{pc}^{-2}$. The effective radii of the galaxies were derived in the $F 160 \mathrm{~W}$ filter with GALFIT (Longhetti et al. 2007) and the stellar masses were estimated by fitting their spectral energy distribution (SED) with the Bruzual and Charlot models using the Chabrier IMF, while visual classification was performed on HST/NIC2 - F160W images (for more details see Sect. 3). We revised the number density estimated by Saracco et al. (2005) considering only the six ETGs at $1.2<z<1.6$ in the field S2F1 $\left(\sim 160\right.$ arcmin $\left.^{2}\right)$, thus, excluding the ETG S2F1-443 at $z=1.91$ (see Sect. 3). We found a comoving number density of ultramassive dense ETGs of $3.8( \pm 1.7) \times 10^{-5} \mathrm{gal} / \mathrm{Mpc}^{-3}$ at $1.2<z<1.6$, which becomes $5.6( \pm 1.9) \times 10^{-5} \mathrm{gal} / \mathrm{Mpc}^{-3}$ considering the spectroscopic incompleteness (a factor $\sim 1.4$ ). The errors on number densities, throughout the paper, are based on Poisson statistics.

GOODS - South sample - Given the small area of the S2F1-MUNICS field, for comparison, and to average the cosmic variance, we also derived the number density of ultramassive dense ETGs in the GOODS-South field covering an area $\left(143 \operatorname{arcmin}^{2}\right)$ comparable to the S2F1 field. To this end, we used the sample of galaxies that are visually classified as ETGs on ACS/F850LP images and studied by Tamburri et al. (2014). The sample contains all of the ETGs in the GOODS-South field that are brighter than $K_{s}=22$ and for masses $M_{\star}>10^{11} M_{\odot}$ is $100 \%$ complete up to $z \sim 2$ (Tamburri et al. 2014). Their stellar masses were derived assuming Bruzual and Charlot models and both the Salpeter IMF and Chabrier IMF. We refer to the latter in our analysis. We found a number density of ultramassive dense ETGs of $1.8( \pm 1.3) \times 10^{-5} \mathrm{gal} / \mathrm{Mpc}^{3}$ in the redshift range $1.3 \lesssim z<1.6$ and of $2.9( \pm 1.6) \times 10^{-5} \mathrm{gal} / \mathrm{Mpc}^{3}$ in the redshift range $0.95<z<1.25$. The structural parameters in the Tamburri et al. sample were derived on the ACS-F850LP images with GALFIT. Hence the selection of ETGs could be biased against dense ETGs because of their larger apparent size $(\sim 30 \%$, Gargiulo et al. 2012) at this wavelength. The number density of dense ETGs does not change, however, even when we correct the effective radius of massive ETGs in the GOODS sample for this difference. In Table 2 and in Fig. 2 we report the measures.

\subsection{The number density of ultramassive dense ETGs at intermediate redshift}

We took advantage of the COSMOS survey to derive the comoving number density of ultramassive dense ETGs at intermediate redshift. We have considered the catalogue of Davies et al. (2015), including all of the available spectroscopic redshifts in the COSMOS area, the catalogue of structural parameters based on the HST/ACS images in the $F 814 W$ filter of Scarlata et al. (2007), and the catalogue including the stellar masses of Ilbert et al. (2013) based on the multiwavelength U1traVISTA photometry. The cross match of these three catalogues produced a sample of 110171 galaxies in the magnitude range $19<F 814 W<24.8$ over the $1.6 \mathrm{deg}^{2}$ of the HST/ACS COSMOS field. We then selected all of the galaxies (25262) that are brighter than $F 814 W<22.5$ mag for which structural parameters and morphological classification are robust (Sargent et al. 2007; Scarlata et al. 2007). We finally extracted our parent sample of 1412 galaxies with stellar mass $M_{*}>10^{11} M_{\odot}$ and earlytype morphology from this sample. We refer to the Zurich Estimator of Structural Types (ZEST) estimates by Scarlata et al. (2007) for the morphological classification. An automated tool for galaxy classification, ZEST uses the Principal Component Analysis to store the full information provided by the entire set of five basic non-parametric diagnostics of galaxy structure 
Table 2. Number density $\rho$ of ultramassive dense $\left(M_{\star}>10^{11} M_{\odot}\right.$, $\Sigma>2500 M_{\odot} \mathrm{pc}^{-2}$ ) ETGs from $z=1.6$ to $\sim 0$.

\begin{tabular}{ccccc}
\hline \hline$z$ & $N$ & $\begin{array}{c}\rho \\
10^{-5} \mathrm{Mpc}^{-3}\end{array}$ & $\begin{array}{c}\sigma_{\rho} \\
10^{-5} \mathrm{Mpc}^{-3}\end{array}$ & Field \\
\hline $0.066-0.1$ & 124 & 0.75 & 0.07 & SDSS \\
$0.2-0.4$ & 13 & 2.6 & 0.9 & COSMOS \\
$0.4-0.6$ & 14 & 1.9 & 0.5 & COSMOS \\
$0.6-0.8$ & 39 & 3.6 & 0.7 & COSMOS \\
$0.8-1.0$ & 79 & 4.7 & 0.8 & COSMOS \\
$0.95-1.25$ & 3 & 2.9 & 1.6 & GOODS-South \\
$1.3-1.6$ & 2 & 1.8 & 1.3 & GOODS-South \\
$1.26-1.6$ & 6 & 3.81 & 1.7 & MUNICS \\
\hline
\end{tabular}

Notes. Column 1: redshift range; Col. 2: number of massive dense ETGs; Col. 3: number density of ultramassive dense ETGs; Col. 4: error on the number density quoted; and Col. 5: field. The number density derived both in the SDSS and in the MUNICS field is a lower limit.

(symmetry, concentration, Gini coefficient, 2nd-order moment of the brightest $20 \%$ of galaxy pixels, and the ellipticity) plus the Sersic index. The software subdivided the galaxies into three main classes: early types (type $=1$ ), disk galaxies (type $=2$ ), and irregular galaxies (type $=3$ ). The robustness of the software was tested both on $\sim 56000$ COSMOS galaxies and on local galaxies that are visually classified, with excellent results. For our analysis, thus, we selected as COSMOS ETGs, all of the galaxies with ZEST-type $=1$. Among these, 551 have a secure spectroscopic redshift (parameter Z_USE $\leq 2$ in the Davies et al. 2014 catalogue), and 145, at $0.2<z<1.0$, have $\Sigma \geq 2500 M_{\odot} \mathrm{pc}^{-2}$. As a further check on morphology, we visually classified the 145 ultramassive dense COSMOS galaxies with ZEST-type $=1$ on the HST/F814W-ACS images (Koekemoer et al. 2007). Actually, we found that all but one are ETGs.

The selection at $F 814 \mathrm{~W}<22.5 \mathrm{mag}$ implies that for $M_{*}>$ $10^{11} M_{\odot}$ the sample is $100 \%$ complete up to $z \sim 0.8(80 \% \mathrm{com}-$ plete to $z \sim 1)$. The stellar masses of Ilbert et al. (2013) are based on the Bruzual and Charlot (2003) models and the Chabrier IMF, which is consistent with our assumptions. The structural parameters of Sargent et al. (2007) are based on a Sersic profile fitting as in our analysis even if performed with GIM2D instead of GALFIT. However, Damjanov et al. (2015) show that the two methods, GIM2D and GALFIT, are in excellent agreement when based on a single Sersic component, as in our case. To derive the number densities of massive ETGs, we applied a correction for the spectroscopic incompleteness that we defined as the ratio between the magnitude distribution (in bin of $0.1 \mathrm{mag}$ ) of the parent sample of ETGs and the sample of ETGs with spectroscopic redshift. In Table 2 we report the comoving number density of ultramassive dense ETGs from $z=0.2$ to $z=1.0$, estimated in bins of redshift of 0.2 (see also Fig. 2). At $z>0.6$, the restframe wavelength sampled by the $F 814 W$ filter is significantly different from that sampled by the filter $F 160 \mathrm{~W}$ at $z=1.5$. As carried out for the GOODS-south data, however, we verified that the number densities derived from the COSMOS data at $z>0.6$ do not change even when we assume a correction of $30 \%$ of the effective radii of galaxies.

\subsection{The number density of ultramassive dense ETGs in local Universe}

As representative sample of local ETGs we chose the dataset analysed by Thomas et al. (2010) ${ }^{1}$. These authors collected a sample of 16502 ETGs extracted from the magnitude-limited

\footnotetext{
1 http://WwW.icg.port.ac.uk/ thomasd/moses.html
}

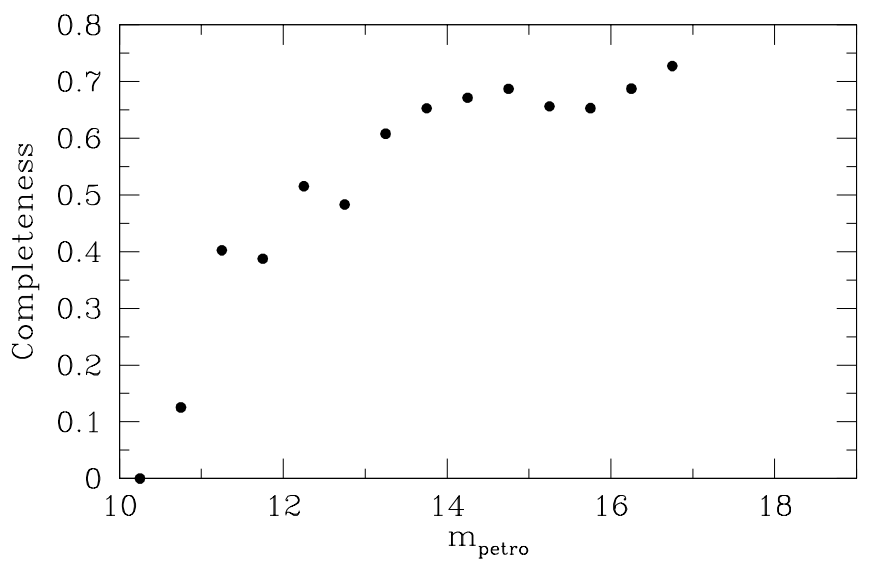

Fig. 1. Completeness of the spectroscopic SDSS galaxy catalogue with respect to the main galaxy sample as a function of galaxy Petrosian magnitude.

sample $(r<16.8)$ of 48023 galaxies selected from the SDSS data release 4 (Adelman-McCarthy et al. 2006) in the redshift range $0.05 \leq z \leq 0.1$. Contrary to the other available samples, as our sample of high- $z$ ETGs, this is the only sample in which ETGs are selected through a visual inspection of SDSS multiband images. The sample is $100 \%$ complete at $M_{\star}>10^{11} M_{\odot}$. We associated with each ETG its stellar mass taken from MPA-JHU catalogue ${ }^{2}$ and obtained through the fit of the multiband photometry (Chabrier IMF), as for higher redshift galaxies. We have adopted as reference the NYU-VAG catalogue (Blanton et al. 2005) for the effective radius. In Appendix A, we discuss the robustness and limits of these estimates.

We bear in mind that the galaxy spectroscopic SDSS survey is biased against dense galaxies (e.g. Strauss et al. 2002; Poggianti et al. 2013a; Taylor et al. 2010b). Briefly, the main galaxy sample (MGS) of galaxy target for the spectroscopic follow-up is composed of all of the galaxies detected at $>5 \sigma$ in the $r$-band with Petrosian magnitude $m_{\text {pet }, r}<17.77$ and $r$-band Petrosian half-light surface brightness $>24.5 \mathrm{mag} \mathrm{arcsec}^{-2}$. On these first cuts, three further selection criteria have been imposed to reject stars, avoid the cross talk, and exclude bright objects that can saturate the CCD. Taylor et al. (2010b) investigated how these cuts affect the distribution of local galaxies in the size mass plane and demonstrated that at $z<0.05$, galaxies with masses and sizes similar to those of $z \sim 2$ galaxies are not targeted for the spectroscopic follow-up. Nonetheless, this incompleteness decreases at increasing redshift: at $z \gtrsim 0.06$, on average, the completeness of the spectroscopic survey for compact massive galaxies is $\sim 80 \%$. For this reason, we selected all of the ultramassive dense ETGs at $0.063<z<0.1$, which amounts 156 galaxies, from the Thomas et al. catalogue. Based on the evidence of Fig. A. 2 we removed from this sample 22 galaxies classified as ultramassive outliers in Appendix A, i.e. galaxies that should not be included in the local ultramassive sample since, most probably, their stellar masses derived through the SED fitting are overestimated. We visually checked this final subsample of 124 galaxies (156-22) and found that no galaxy has a close companion, is in the halo of a bright star, or is involved in a merger; these factors that can alter the estimate of the effective radius. However, we noted that $\sim 15 \%$ of the sample (20 galaxies) does not have a clear elliptical or S0 morphology, even though they are included in the Thomas et al. ETG sample. We checked

\footnotetext{
2 http://www . mpa-garching.mpg.de/SDSS/DR7/
} 


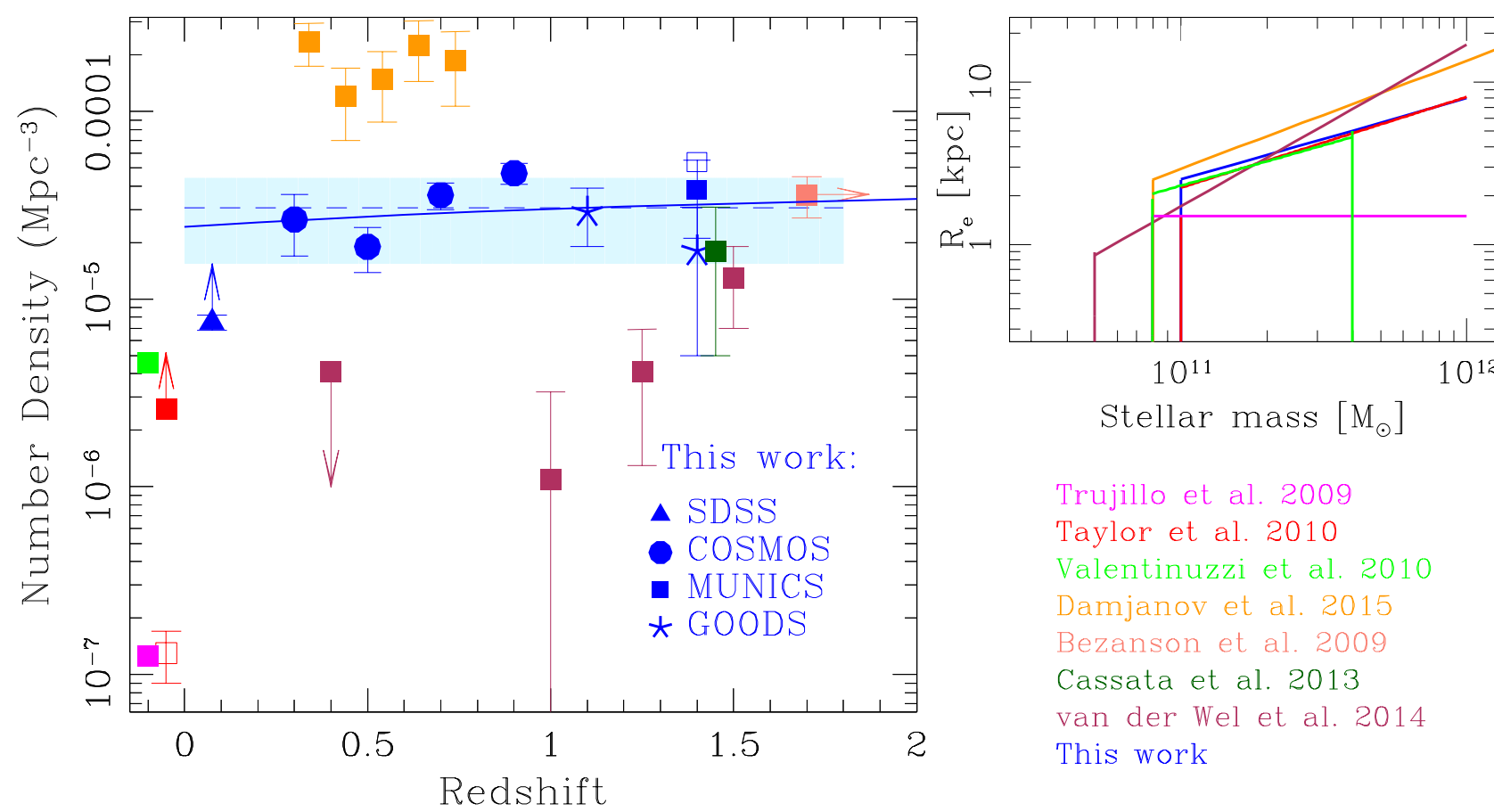

Fig. 2. Left: evolution of the comoving number density of ultramassive dense ETGs $\left(M_{\star}>10^{11} M_{\odot}, \Sigma>2500 M_{\odot} \mathrm{pc}^{-2}\right)$ from redshift 1.6 to redshift $\sim 0$ (blue symbols). The open blue square indicates the number density of ultramassive dense MUNICS ETGs at $\langle z\rangle=1.4$ corrected for the spectroscopic incompleteness of the MUNICS survey (see text). Solid blue line is the best-fit relation to our data in the range $0.066<z<1.6$ $\left(\rho \propto(1+z)^{0.3}\right)$, while the blue dotted line indicates the mean value over the redshift range $0.066<z<1.6$ (the dashed area indicates the $1 \sigma$ deviation around this value). The orange squares indicate the number density for ultramassive compact galaxies by Damjanov et al. (2015), the magenta square by Trujillo et al. (2009), the light green square by Valentinuzzi et al. (2010), the purple squares by van der Wel et al. (2014), the salmon squares by Bezanson et al. (2009), the dark green square by Cassata et al. (2013), and the open red square by Taylor et al. (2010a). The points by Taylor et al. (open red square) and Cassata et al. (dark green square) are derived selecting from their sample only the galaxies with $M_{\star}>10^{11} M_{\odot}$ (see Table 3 ). The filled red square is the number density by Taylor et al. obtained using the circularized Sersic $R_{\mathrm{e}}$. Right: cuts in $R_{\mathrm{e}}$ and $M_{\star}$ of our sample and other samples in the size to mass plane (colours are the same in the left panel).

the structural parameters of these galaxies and they have a mean Sersic index $n \simeq 3.5$ (for all of the galaxies $n>2.5$ ) that is consistent with the light profile of a spheroidal galaxy, but an axis ratio of $b / a<0.4$. Since the Blanton catalogue does not provide an axis ratio, we used that obtained from the SDSS pipeline, fitting the real galaxies with a de Vaucouleur profile. Actually, the $b / a$ should be not dependent on the surface brightness law used to fit the profile. We left these elongated objects in the final sample, since the Thomas et al. sample is widely adopted and studied, but in Sect. 2.4 we show that this choice does not impact our results.

Aside from this source of incompleteness due to the target selection, we have to consider that not all of the spectroscopic targets were actually observed (for example because two fibres cannot be placed closer than $55^{\prime \prime}$ on a given plate). This source of incompleteness should be more important for the brightest galaxies and should be taken into account in the derivation of the comoving number density. To quantify this incompleteness we extracted the MGS from the DR4 database restricting the query to only the sciencePrimary objects in order to reject multiple observations (648 852 galaxies). From this sample, we rejected galaxies classified as quasars leaving with 636980 galaxies. Among these objects, $384564(\sim 60 \%)$ have been spectroscopically observed and $\sim 88 \%$ of them have secure redshifts (i.e. zwarning $=0$ ). We defined the completeness in bins of magnitude as the ratio of galaxies with secure redshift over the number of galaxies in the MGS (see Fig. 1). Taking this in consideration, we found a number density for local ultramassive dense ETGs of $0.75( \pm 0.07) \times 10^{-5} \mathrm{gal} / \mathrm{Mpc}^{-3}$. We repeated the measure of $\rho$, assuming as stellar masses for local ETGs those derived by Kauffmann et al. (2003) with spectral indices (see Appendix A) and we found $\rho=0.7( \pm 0.06) \times 10^{-5} \mathrm{gal} / \mathrm{Mpc}^{-3}$. We corrected the number density for the incompleteness of the spectroscopic sample with respect to the MGS but the original selection cuts of the MGS can exclude part of massive dense ETGs especially in the lowest redshift bin. The number density we quote is therefore a lower limit.

\subsection{The number density evolution of ultramassive dense ETGs over the last 9 Gyr: results}

Figure 2 shows the evolution of number density for the ultramassive dense ETGs in the last $\sim 9$ Gyr. Once the possible sources of non-homogeneity are taken into account the mean value of the number density of ultramassive dense ETGs, within the uncertainties, is $\sim 3.0 \pm 1.5 \times 10^{-5} \mathrm{Mpc}^{-3}$. We observe that at $z \sim 0.8$, in the COSMOS field, there is a significant overdensity (see e.g. Damjanov et al. 2015, and reference therein) and that, correspondingly, this is the point that most deviates in Fig. 2. We fitted the number densities estimated in the redshift range $0.06 \lesssim z<1.6$ with a power law, treating the SDSS and MUNICS point as lower limits, and found $\rho(z) \propto(1+z)^{0.3 \pm 0.8}$, showing that the number density of ultramassive dense ETGs may decrease from $z=1.6$ to $z=0$, approximately by a factor 1.3 . We repeated the fit excluding from local sample those galaxies with ambiguous morphology (see Sect. 2.3), but the slope does not change, since in the fit this point is considered a lower limit. 
Table 3. Quick comparison of the main characteristics of our sample with respect to those by other authors we compare (see text and Fig. 2).

\begin{tabular}{|c|c|c|c|c|}
\hline Reference & $z$ & Stellar mass range & Compactness definition & Supplementary galaxy selections \\
\hline This work & $0 \lesssim z \lesssim 1.6$ & $M_{\star}>10^{11}$ & $\Sigma \geq 2500 M_{\odot} \mathrm{pc}^{-2}$ & $\begin{array}{l}\text { Elliptical morphology } \\
\text { (Visual classification) }\end{array}$ \\
\hline Trujillo et al. (2009) & $0<z<0.2$ & $M_{\star}>8 \times 10^{10}$ & $R_{\mathrm{e}}<1.5 \mathrm{kpc}$ & - \\
\hline Taylor et al. (2010) & $0.066<z<0.12$ & $M_{\star}>10^{11}$ & $\begin{array}{c}\log \left(R_{\mathrm{e}, z} / \mathrm{kpc}\right)< \\
0.56 \times\left(\log \left(M_{\star} / M_{\odot}\right)-9.84\right)-0.3\end{array}$ & $(u-r)>2.5$ \\
\hline Valentinuzzi et al. (2010) & $0.04<z<0.07$ & $8 \times 10^{10}<M_{\star}<4 \times 10^{11} M_{\odot}$ & $\Sigma>3000 M_{\odot} \mathrm{pc}^{-2}$ & - \\
\hline Damjanov et al. (2015) & $0.2<z<0.8$ & $M_{\star}>8 \times 10^{10}$ & $\begin{array}{c}\log \left(R_{\mathrm{e}} / \mathrm{kpc}\right)< \\
0.568 \times \log \left(M_{\star} / M_{\odot}\right)-5.74\end{array}$ & NUVrJ colours \\
\hline Bezanson et al. (2009) & $z=2.5$ & $M_{\star}>10^{11}$ & - & Quiescent \\
\hline Cassata et al. (2013) & $1.2<z<1.6$ & $M_{\star}>10^{11}$ & $\Sigma \geq 2500 M_{\odot} \mathrm{pc}^{-2}$ & $\begin{aligned} & s S F R<10^{-11} \mathrm{yr}^{-1} \\
+ & \text { elliptical morphology }\end{aligned}$ \\
\hline van der Wel et al. (2014) & $0 \lesssim z \lesssim 1.6$ & $M_{\star}>5 \times 10^{10}$ & $R_{\mathrm{e}} /\left(M_{\star} / 10^{11} M_{\odot}\right)^{0.75}<1.5 \mathrm{kpc}$ & UVJ red colours \\
\hline
\end{tabular}

Notes. Column 1: reference paper; Col. 2: redshift range; Col. 3: stellar mass limits (for Taylor et al. and Cassata et al. is reported the one we use); Col. 4: compactness definition; Col. 5: other supplementary selections applied to the samples.

\subsection{Comparison with previous works}

The number density estimates of dense ETGs are clearly dependent on many factors, such as the stellar mass limit, the definition of compact galaxies, and the criteria adopted to select the sample (e.g. visual classification, colour, and passivity). This implies that any comparison of our results with those by other authors is not straightforward. In the following we try to compare our estimates of number density with those by other authors that adopted selection criteria as similar as possible to ours. Given the many factors concurring in the final estimate of $\rho$, in Fig. 2 we report, in the size mass plane, the stellar mass and effective radius limits of each sample to better visualize these comparisons. At the same time, Table 3 immediately shows the characteristics of all of the samples studied.

\subsubsection{Local Universe}

For what concerns the local Universe, there is a clear tension among the results already published, since the number densities derived from the SDSS database (Trujillo et al. 2009; Taylor et al. 2010a) turn out to be at least 1-2 order of magnitude lower than those derived from other databases (e.g. WINGS/PM2GC; Valentinuzzi et al. 2010; Poggianti et al. 2013b, see Fig. 2). Interestingly, Fig. 2 shows that our estimate of local number density, although derived from the SDSS database, is much more in agreement with the finding of Valentinuzzi et al. (2010) than with that by Trujillo et al. (2009) and Taylor et al. (2010a). In the following, we investigate the possible origins of the observed discrepancy.

In fact, Taylor et al. (2010a) search for counterparts of high$z$ compact massive galaxies in the DR7 SDSS spectroscopic database. To this aim, they select from the sample of local galaxies at $0.066<z<0.12$, with $M_{\star}>10^{10.7} M_{\odot}$, and $\log \left(R_{\mathrm{e}, z} / \mathrm{kpc}\right)<0.56 \times\left(\log \left(M_{\star} / M_{\odot}\right)-9.84\right)-0.3$, i.e. with radius in the SDSS- $z$ band at least twice as small as the local SMR by Shen et al. (2003), those with ${ }^{0.1}(u-r)>2.5^{3}$ to select the descendant candidates of high- $z$ dense galaxies. They reject from their sample all of the galaxies with possible problems with their size measurement (due to confusion with, e.g.

\footnotetext{
3 The superscript 0.1 indicates rest-frame photometry redshifted to $z=0.1$, (see, e.g., Blanton \& Roweis 2007).
}

other galaxies, with extended halos, and/or with spikes of bright stars) or mass estimate, for a total final sample of 63 galaxies. Among these galaxies, 11 have stellar mass $>10^{11} M_{\odot}$ and yield a number density of $1.3( \pm 0.4) 10^{-7} \mathrm{gal} / \mathrm{Mpc}^{3}$ over the entire volume of the SDSS-DR7 spectroscopic survey (see Fig. 2 open red square). This value, which is not corrected for the spectroscopic incompleteness of SDSS-DR7 survey, is two orders of magnitude lower than the value we found. Actually, this huge observed difference cannot only be ascribed to the completeness function. In fact, it is really difficult to pinpoint the origin of such a difference between our result and their result, since the two samples are selected differently.

In fact, the cut they apply in $R_{\mathrm{e}}$ is very similar to our cut in $\Sigma$ (see size mass plane in Fig. 2), however, unlike us, they select only red galaxies. To test the impact of the colour selection, we computed the ${ }^{0.1}(u-r)$ colour for the galaxies of our sample and found that all but one have ${ }^{0.1}(u-r)>2.5$, so the colour selection cannot be the source of the observed difference in the number density. We then investigated the impact on the number density of the different $R_{\mathrm{e}}$ used. Actually, we use the circularized $R_{\mathrm{e}}$ obtained fitting the surface brightness profile with a Sersic law (hereafter $R_{\mathrm{e}, \mathrm{Ser}}$ ), instead of the semi-major axis $\mathrm{SM}_{\mathrm{e}}$, used by Taylor et al., obtained from SDSS pipeline fitting the SDSS images with a de Vaucouleur profile (hereafter $\mathrm{SM}_{\mathrm{e}, \mathrm{d} V}$ ). For this reason, we repeated their analysis looking for differences in the $\rho$ estimates varying the definition of $R_{\mathrm{e}}$. To do this, we selected the 226312 galaxies with secure redshift (i.e. zwarning $=0$ ) and spectroscopic redshift $0.066<z_{\text {spec }}<0.12$ from the primary observations of the DR7 spectroscopic sample. Among these, we collected those with $M_{\star}>10^{11} M_{\odot}, \log \left(\mathrm{SM}_{\mathrm{e}, \mathrm{d} V} / \mathrm{kpc}\right)<0.56 \times\left(\log \left(M_{\star} / M_{\odot}\right)\right.$ $-9.84)-0.3$, and ${ }^{0.1}(u-r)>2.5$. For the $\mathrm{SM}_{\mathrm{e}, \mathrm{d} V}$ we retrive the catalogue from the SDSS database, consistently with Taylor et al., while for the stellar masses derived through the SED fitting, we refer to the MPA-JHU catalogue (see Sect. 2.3). We visually inspected this subsample, and we rejected all of the galaxies with problems related to the size measurement (e.g. merging galaxies, or close pairs or galaxies in the halo of a bright stars), leaving us with 49 galaxies. None of these galaxies is a quasar. To check the reliability of the stellar mass estimates, we applied the same procedure as in Appendix A. Among the 49 galaxies of our selection, 38 have a stellar mass estimate that is also derived from the spectral indices (stellar masses 
from spectral indices were only derived for galaxies in the DR4 database), and for ten of these ( $26 \%)$, the two estimates are consistent. These ten galaxies are all included in the subsample of 11 massive galaxies of Taylor and collaborators. Assuming that the fraction of galaxies with consistent estimates of $M_{\star}$ is constant, we should expect $\sim 12$ galaxies $(49 \times 0.26)$ with $M_{\star}>10^{11} M_{\odot}, \log \left(\mathrm{SM}_{\mathrm{e}, \mathrm{d} V} / \mathrm{kpc}\right)<0.56 \times\left(\log \left(M_{\star} / M_{\odot}\right)-9.84\right)$ $-0.3,{ }^{0.1}(u-r)>2.5$ in DR7 release, in agreement with Taylor and collaborators. When circularized SDSS de Vaucouleur radii $\left(R_{\mathrm{e}, \mathrm{d} V}\right)$ are used instead, we found 569 galaxies with $M_{\star}>$ $10^{11} M_{\odot}, \log \left(R_{\mathrm{e}, \mathrm{d} V} / \mathrm{kpc}\right)<0.56 \times\left(\log \left(M_{\star} / M_{\odot}\right)-9.84\right)-0.3$, ${ }^{0.1}(u-r)>2.5$, among which 186 have good $R_{\mathrm{e}}$ estimates and stellar mass, against the 12 found when semi-major axes are used. Finally, when we use the Blanton $R_{\mathrm{e}, \mathrm{Ser}}$ (circularized and obtained fitting a Sersic profile), we find 687 galaxies with $M_{\star}>10^{11} M_{\odot}, \log \left(R_{\mathrm{e}, \mathrm{Ser}} / \mathrm{kpc}\right)<0.56 \times\left(\log \left(M_{\star} / M_{\odot}\right)-9.84\right)$ -0.3 , and ${ }^{0.1}(u-r)>2.5$, which after all of the checks and visual inspection, result in a final sample of $\sim 224$ galaxies, which is a factor $>20$ higher than that obtained using the semi-major axis. The higher number of galaxies we have found using $R_{\mathrm{e}, \mathrm{Ser}}$ with respect to the one obtained using circularized de Vaucouleur radius is consistent with the expectations. Compact galaxies are known to generally have Sersic index $n<4$, thus, the value of $R_{\mathrm{e}}$ obtained fitting a de Vaucouleur profile should be higher with respect to that obtained fitting a Sersic law. This implies that the number of compact objects, which is defined using a Sersic- $R_{\mathrm{e}}$ should be greater than that obtained using a de Vaucouleur- $R_{\mathrm{e}}$, as we find. For more details on the comparison of the local samples selected using circularized $R_{\mathrm{e}}$ and semi-major axis $\left(\mathrm{SM}_{\mathrm{e}}\right)$, or de Vaucouleurs and Sersic $R_{\mathrm{e}}$ see Appendix B. Thus, these checks show that the much lower number density found by Taylor et al. with respect to our estimate is because that they use the semimajor axis. Once the circularized Sersic $R_{\mathrm{e}}$ values are used, the number density (not corrected for spectroscopic incompleteness) increases up to $2.6( \pm 0.2) 10^{-6} \mathrm{gal} / \mathrm{Mpc}^{-3}$ and is qualitatively in agreement with our estimate.

Trujillo et al. (2009), from the analysis of the SDSS DR6 database, claim that only the $0.03 \%$ of galaxies at $z<0.2$ with $M_{\star}>8 \times 10^{10} M_{\odot}$ have $0.05^{\prime \prime}<R_{\mathrm{e}}<1.5 \mathrm{kpc}$. We found that among the 99783 galaxies in the SDSS DR4 spectroscopic sample with $z<0.2$, zwarning $=0$ and $M_{\star}>8 \times 10^{10} M_{\odot}$, only 113 (i.e. $\sim 0.11 \%$ of the massive sample) have $0.05^{\prime \prime}<R_{\mathrm{e}}<1.5 \mathrm{kpc}$. The visual inspection rejects 76 galaxies, for example in the halo of a bright star or a merger galaxy. This leaves a sample of 37 galaxies, i.e. the $0.037 \%$ (versus $0.03 \%$ by Trujillo) of the original sample of local galaxies with $M_{\star}>8 \times 10^{10} M_{\odot}$. In this case, thus, the difference we found between our estimate and that by Trujillo et al. is totally ascribed to the different selection criteria. The authors probe a region of the size-mass plane that is included in our region (see size mass plane in Fig. 2) and known to be highly incomplete in the SDSS spectroscopic survey. Both factors lead to a very low number density.

Valentinuzzi et al. (2010), studying the sample of 78 clusters included in the WINGS survey, have derived the number density of local $(0.04<z<0.07)$ galaxies with $8 \times 10^{10}<$ $M_{\star}<4 \times 10^{11} M_{\odot}$ and $\Sigma>3000 M_{\odot} \mathrm{pc}^{-2}$. Assuming there are no dense massive galaxies outside the clusters, they found a hard lower limit to the number density of these systems of $0.46 \times 10^{-5} \mathrm{gal} / \mathrm{Mpc}^{-3}$. Using the DR7, we found 181 galaxies in the same $z / M_{\star} / \Sigma_{g}$ range, where $\Sigma_{g}$ is the mean stellar mass density derived using the effective radius measured by Blanton on SDSS $g$-band images, i.e. the SDSS band that is closer to the $v$-band used by Valentinuzzi et al. (2010). Among the 181 selected galaxies, 120 have reliable structural parameters, and $\sim 64 \%$ of them has a robust stellar mass estimate. Taking the effect of the incompleteness into account, we found that the number density is $\rho=5.21 \times 10^{-5} \mathrm{gal} / \mathrm{Mpc}^{3}$, thus a factor 10 higher than the lower limit of Valentinuzzi et al. (2010). Our estimate is in the right direction with respect to that of Valentinuzzi, but we are not in the position to make a more quantitative comparison. We avoid comparing our results with other analyses (e.g. Poggianti et al. 2013b) which, although treating the evolution of the number density of compact galaxies, adopt a much lower cut in stellar mass with respect to our cut (e.g. $\sim 1-5 \times 10^{10} M_{\odot}$ ).

\subsubsection{Intermediate redshift range}

Carollo et al. (2013) derive the number density of $M_{\star}>10^{11} M_{\odot}$ quenched $\left(s S F R<10^{11} \mathrm{yr}^{-1}\right)$ and elliptical galaxies with $R_{\mathrm{e}}<$ $2.5 \mathrm{kpc}$ over the redshift range $0.2<z<1$ and find that it decreases by a factor $\sim 30 \%$. Their analysis is focused on a sample of galaxies, on average, denser than the one studied here, and differently from us they do not consider all the elliptical galaxies, but the subsample of passive ones. Despite this, their trend is in fair agreement with our findings. In fact, we find a decrease of $\rho$ of $\sim 20 \%$ in the redshift range $0.2<z<1.0$.

Damjanov et al. (2015) present the number density of quiescent (colour-colour selected) galaxies with $\log \left(M_{\star} / R_{\mathrm{e}}^{1.5}\right)>$ $10.3 M_{\odot} \mathrm{kpc}^{-1.5}$ and $M_{\star}>8 \times 10^{10}$ in the COSMOS field and find that it is roughly constant in the redshift range $0.2<z<0.8$. Their sample includes our one, thus, as expected their number density values are higher than those we find, but once again, their trend of $\rho$ with $\mathrm{z}$ is in fair agreement with us. Actually, from $z=0.8$ to $z=0.2$ we find that $\rho$ decreases by $\sim 10 \%$.

Finally, Tortora et al. (2016) derived the number density of massive $\left(M_{\star}>8 \times 10^{10} M_{\odot}\right)$, supercompact $\left(R_{\mathrm{e}}<1.5 \mathrm{kpc}\right)$ elliptical galaxies they detected in the Kilo Degree Survey. Although their compactness criterion is more restrictive than our criterion, the authors found an almost constant trend in the redshift range $0.2 \lesssim z \lesssim 0.6$, consistently with our results.

\subsubsection{High redshift}

Bezanson et al. (2009), integrating the Schechter mass function given in Marchesini et al. (2009), find that the number density of the galaxies with $M_{\star}>10^{11} M_{\odot}$ is $7.2_{-1.1}^{+1.1} \times 10^{-5} \mathrm{Mpc}^{-3}$ at $z=2.5$. Assuming that approximately half of the galaxies with $M_{\star}>10^{11} M_{\odot}$ are compact, they find that the number density of compact massive galaxies at $z=2.5$ is $3.6_{-0.9}^{+0.9} \times 10^{-5} \mathrm{Mpc}^{-3}$. The authors assume the Kroupa (2001) IMF, which provides stellar masses a factor $\sim 1.2$ higher than that obtained assuming the Chabrier IMF, so their value is an upper limit with respect to our values. The estimate by Bezanson et al. (2009) at $z=2.5$ is in agreement with the mean value we find at $z<1.6$ and with our best fit.

Cassata et al. (2013) derive the number density of compact (i.e. $1 \sigma$ below the local SMR) and ultracompact (more than 0.4 dex smaller than local counterparts of the same mass) passive $\left(s S F R<10^{-11} \mathrm{yr}^{-1}\right)$ galaxies with elliptical morphology and $M_{\star}>10^{10} M_{\odot}$ in the $\sim 120 \operatorname{arcmin}^{2}$ of the GOODS-South field covered by the first four CANDELS observations (Grogin et al. 2011; Koekemoer et al. 2011) and by the Early Release Science Program 2 (Windhorst et al. 2011). Using their published sample of 107 galaxies, we selected the only two galaxies with $M_{\star}>$ $10^{11} M_{\odot}$ and $\Sigma>2500 M_{\odot} \mathrm{pc}^{-2}$ at $1.2 \leqslant z \leqslant 1.6$, which account 
for a number density of $1.47( \pm 1.04) 10^{-5} \mathrm{gal} / \mathrm{Mpc}^{3}$. This value is slightly lower than the value we found in the GOODS - South field (see Table 1), nonetheless we bear in mind that the authors selected passive and elliptical galaxies while we apply a pure visual classification. In comparing the sample of passive galaxies with that of elliptical galaxies that were visually classified, in the GOODS - South field, Tamburri et al. (2014) have shown that about $\sim 26 \%$ of ETGs have $s S F R>=10^{-11} \mathrm{yr}^{-1}$, i.e. are not passive. Taking this into consideration, we corrected the number density by a factor 1.26 , obtaining $1.85( \pm 1.30) \times 10^{-5} \mathrm{gal} / \mathrm{Mpc}^{3}$, which is in agreement with our estimate on the GOODS - South field. The agreement between our number density estimate and that from the Cassata sample, although on the same field, is not straightforward. Actually, we derived all of the quantities involved in our estimate (e.g. stellar mass and effective radius) independently. Despite the different approaches and procedures, the number densities are consistent, thereby reinforcing the robustness of the result.

In the 3D-HST survey, van der Wel et al. (2014) derive the number density of compact $\left(R_{\mathrm{e}} /\left(M_{\star} / 10^{11} M_{\odot}\right)^{0.75}<1.5 \mathrm{kpc}\right)$ passive (on the basis of their UVJ colours) galaxies with $M_{\star}>$ $5 \times 10^{10} M_{\odot}$ in the redshit range $0<z<3$. In contrast with our findings, the authors find a decrease by a factor $\gtrsim 10$ from $z=1.6$ to $z=0$. We have to premise that a reliable comparison with their work is unfeasible given the much different criteria used to select the samples, so we cannot go deeper into the orgin of the drop, which could be due, for example, to the different stellar mass limit $\left(M_{\star}>5 \times 10^{10} M_{\odot}\right.$ vs. $\left.M_{\star}>10^{11} M_{\odot}\right)$ and/or to a peculiarity of the population of red galaxies; in principle, we have no reason to suppose that the formation and evolutionary picture of ultramassive dense ETGs is similar to that of less massive and red galaxies. We find it jarring, however, that the values they find are a factor $>5$ lower than ours, despite their more relaxed cut in stellar mass. In the following we simply try to understand the origin of this discepancy. The criterion they adopted to select compact galaxies is very similar to our criterion (see Fig. 2), along with the reference rest-frame wavelength for the $R_{\mathrm{e}}$ ( $r$-band for us and $5000 \AA$ for them). Thus, the reasons for the huge discrepancy we observe cannot reside in these features. There are two main differences between our approaches. First, they used the semi-major axes $\mathrm{SM}_{\mathrm{e}}$ instead of the $R_{\mathrm{e}}$. Second, they did not directly measure the semi-major axis on images sampling the $5000 \AA$ rest frame at the various redshift considered, but they derived it from the $\mathrm{SM}_{\mathrm{e}, F 125 W}$ (i.e. the semi-major axis measured on the $F 125 \mathrm{~W}$-band images) assuming a variation with $\lambda\left(\Delta \log \mathrm{SM}_{\mathrm{e}} / \Delta \log \lambda\right)=-0.25$ (see their Eq. (2)). In order to investigate whether one of these aspects (or both) are responsible for the observed discrepancy, we used our sample of ETGs selected in the GOODS-South field by Tamburri et al. (2014) and derived the number densities of ultramassive dense ETGs following the recipe by van der Wel et al. (2014). In details, we associated the structural parameters derived by van der Wel in both the $F 125 \mathrm{~W}$ and $F 160 \mathrm{~W}$ filter with each galaxy of our sample. From these we estimated the $R_{\mathrm{e}, 5000} \AA$ der, and $\mathrm{SM}_{\mathrm{e}, 5000} \AA \mathrm{der}$, following their Eq. (2). In the redshift range $0.95<z<1.25$ in the GOODS South field we found three galaxies with $M_{\star} \geq 10^{11} M_{\odot}$ and $\Sigma \geq 2500 M_{\odot} \mathrm{pc}^{-2}$ (see Table 2), while there are no ETGs using $R_{\mathrm{e}, 5000} \AA$ der and $\mathrm{SM}_{\mathrm{e}, 5000} \AA$ der. At $1.3<z<1.6$ we found two galaxies. We found the same number of objects using $R_{\mathrm{e}, 5000} \AA$ der' and just one using the semi-major axis $\mathrm{SM}_{\mathrm{e}, 5000} \AA$ der. Thus, applying the same procedure by van der Wel et al. to derive the number density of ultramassive dense ETGs we find values of $\rho$ lower or comparable (and no higher) than those presented in their paper; hence this is much more consistent with the expectations, considering the fact that our cut in stellar mass is more restrictive (for a more exaustive analysis on the effect of the extrapolation of the $\mathrm{SM}_{\mathrm{e}}$ using the van der Wel et al. Eq. (2), see Appendix C). However, we should keep in mind that in this comparison both the cosmic variance, and also the different selection of the sample (elliptical versus passive) have a strong effect on the number density estimates. All of these checks highlight the huge impact of the criteria and quantities used in each analysis on the comparison of the results. A fair comparison is really hard to address and this stresses the impelling necessity of analysis on homogeneous samples over cosmic time to draw firm conclusions.

\section{The evolution of structural and dynamical parameters of ultramassive dense ETGs}

The mild evolution in the number density shows that in the local Universe, the ultramassive dense ETGs are at most $\sim 25 \%$ less numerous than at $\langle z\rangle=1.4$. Thus, if a significant portion of ultramassive dense ETGs evolves in size, new ETGs have to appear at lower redshift to maintain their number density as almost constant. The observed mild number density evolution also opens to the possibility that most $(\sim 75 \%)$ of the ultramassive dense ETGs passively evolve from $\langle z\rangle=1.4$ to $z=0$. In this case, local and high- $z$ ultramassive dense ETGs should also share the same structural and dynamical properties. In the following we compare the structural $\left(R_{\mathrm{e}}\right.$ and $\left.M_{\star}\right)$ and dynamical properties $\left(\sigma_{\mathrm{e}}\right)$ of the ultramassive dense ETGs over the last 9 Gyr. We highlight that in this second part of analysis, we investigate the same class of objects studied in Sect. 2, that is ETGs visually classified with $M_{\star}>10^{11} M_{\odot}$ and $\Sigma>2500 M_{\odot} \mathrm{pc}^{-2}$. The request of available velocity dispersion, however, does not allow us to use the same samples presented in Sect. 2. In Sect. 3.1 we present the samples used for this part of the analysis.

\subsection{The comparison samples}

In order to compare the structural and dynamical properties of ultramassive dense ETGs over the last 9 Gyr, we still refer, as local reference, to the sample of 124 ETGs presented in Sect. 2.3; this section also provides the stellar mass and effective radius used. For the velocity dispersions we referred to DR7 $\sigma$ since in DR7 there have been improvements to the algorithms that photometrically calibrate the spectra and all spectra were rereduced (see Thomas et al. 2013 for more tests on the reliability of DR7 $\sigma$ estimates).

For the intermediate and high- $z$ sample, we cannot refer to the same samples used to estimate the number density in Sect. 2, since not all of the ETGs have available velocity dispersions. We refer to the sample of field and cluster ETGs published by Saglia et al. (2010) to probe the intermediate redshift range $(0.2 \lesssim z \lesssim 0.9)$. The authors selected the galaxies of their sample on the basis of their spectra (requesting the total absence or the presence of only weak [OII] lines), and they also provided the morphological classification (T type) for those with HST images. We selected from their sample, the galaxies with elliptical morphology ( $\mathrm{T}$ type $\leqslant-4$ ) and with $M_{\star}>10^{11} M_{\odot}$ and $\Sigma>2500 M_{\odot} \mathrm{pc}^{-2}$. The stellar mass provided by the authors are derived adopting a diet Salpeter IMF. We converted these estimates to Chabrier IMF, scaling the masses by 0.085 dex. For the majority of the galaxies, the structural parameters were derived from the $F 814 \mathrm{~W}$-band images, while $I$-band VLT images were used for a small fraction of galaxies. We integrated the 
Table 4. Sample of high- $z$ ETGs collected from literature.

\begin{tabular}{|c|c|c|c|c|c|c|c|c|c|c|}
\hline Object & $\begin{array}{c}\text { RA } \\
\text { h:m:s }\end{array}$ & $\begin{array}{c}\text { Dec } \\
\mathrm{d}: \mathrm{m}: \mathrm{s}\end{array}$ & $z$ & $\begin{array}{c}R_{\mathrm{e}} \\
(\mathrm{kpc})\end{array}$ & $n$ & $b / a$ & Camera-Filter & $\begin{array}{c}\sigma \\
\left(\mathrm{km} \mathrm{s}^{-1}\right)\end{array}$ & $\begin{array}{c}\sigma_{\mathrm{e}} \\
\left(\mathrm{km} \mathrm{s}^{-1}\right)\end{array}$ & $\begin{array}{c}\log M_{\star} \\
\left(M_{\odot}\right)\end{array}$ \\
\hline $\mathrm{A} 17300(1)$ & $14: 18: 39.78$ & $52: 41: 59.51$ & 1.423 & $2.9 \pm 0.51(2)$ & 5.3 & $\ldots$ & HST/WFC3-F160W & $265 \pm 7$ & $272 \pm 7$ & 11.26 \\
\hline A21129(1) & $14: 19: 10.56$ & $52: 46: 26.79$ & 1.583 & $1.5 \pm 0.27(2)$ & 5.0 & . & HST/WFC3-F160W & $260 \pm 9$ & $278 \pm 10$ & 11.18 \\
\hline $\mathrm{C} 21434(1)$ & 10:00:36.10 & $2: 32: 13.77$ & 1.522 & $1.9 \pm 0.33(2)$ & 3.1 & $\ldots$ & HST/WFC3-F160W & $218 \pm 16$ & $230 \pm 17$ & 11.20 \\
\hline C20866(1) & $9: 59: 18.64$ & $2: 31: 39.03$ & 1.522 & $2.4 \pm 0.42(2)$ & 3.0 & $\ldots$ & HST/WFC3-F160W & $272 \pm 23$ & $282 \pm 23$ & 11.30 \\
\hline $21750(3)$ & 215.03490 & 52.9829 & 1.242 & $2.59 \pm 0.26$ & 5.2 & 0.57 & HST/WFC3-F160W & $\ldots$ & $259 \pm 16$ & 11.03 \\
\hline $7310(3)$ & 150.05791 & 2.2904 & 1.255 & $4.34 \pm 0.43$ & 3.8 & 0.87 & HST/WFC3-F160W & $\ldots$ & $167 \pm 15$ & 11.13 \\
\hline $\mathrm{S} 2 \mathrm{~F} 1-142(4)$ & $03: 06: 36.51$ & 00:03:01.0 & 1.386 & $3.04 \pm 0.12$ & 3.5 & 0.74 & HST/NIC2-F160W & $340_{-60}^{120}$ & $347_{-60}^{120}$ & 11.54 \\
\hline
\end{tabular}

Notes. Column 1: ID; Col. 2: RA; Col. 3: Dec; Col. 4; redshift; Col. 5: circularized effective radius; Col. 6: Sersic index; Col. 7: axis ratio; Col. 8: filter adopted to derive the surface brightness parameters; Col. 9: measured velocity dispersion; Col. 10: velocity dispersion within $R_{\mathrm{e}}$; Col. 11: stellar masses. References for the velocity dispersion values are listed near the ID value. When a data of the galaxy comes from a paper other than that in which the $\sigma$ is published, the new reference is specified near its value.

References. (1) Bezanson et al. (2013); (2) van de Sande et al. (2013); (3) Belli et al. (2014); (4) Longhetti et al. (2014).

Table 5. Our sample of high-z ETGs.

\begin{tabular}{|c|c|c|c|c|c|c|c|c|c|c|c|}
\hline Object & RA & Dec & $z$ & $\begin{array}{c}R_{\mathrm{e}} \\
(\mathrm{kpc})\end{array}$ & $n$ & $b / a$ & $\begin{array}{c}\sigma \\
\left(\mathrm{km} \mathrm{s}^{-1}\right)\end{array}$ & $\begin{array}{c}\sigma_{\mathrm{off}} \\
\left(\mathrm{km} \mathrm{s}^{-1}\right)\end{array}$ & $\begin{array}{c}\sigma_{\mathrm{e}} \\
\left(\mathrm{km} \mathrm{s}^{-1}\right)\end{array}$ & $\log \left(M_{\star, \mathrm{cha}} / M_{\odot}\right)$ & $K$ \\
\hline $\mathrm{S} 2 \mathrm{~F} 1-511$ & 03:06:34.04 & $+00: 02: 30.9$ & 1.267 & $2.09 \pm 0.07$ & 3.3 & 0.60 & 269 & {$[-4 \div 12]$} & $281_{-40}^{+26}$ & 11.05 & $18.1 \pm 0.1$ \\
\hline S2F1-633 & $03: 06: 35.10$ & $+00: 04: 43.6$ & 1.297 & $2.59 \pm 0.11$ & 4.1 & 0.53 & 434 & {$[-24 \div-7]$} & $447_{-19}^{+14}$ & 11.48 & $18.2 \pm 0.1$ \\
\hline S2F1-527 & 03:06:43.34 & $+00: 02: 44.8$ & 1.331 & $1.57 \pm 0.24$ & 3.1 & 0.88 & 226 & {$[-16 \div 1]$} & $240_{-28}^{+25}$ & 11.14 & $18.3 \pm 0.1$ \\
\hline $\mathrm{S} 2 \mathrm{~F} 1-389$ & $03: 06: 28.03$ & $+00: 00: 31.6$ & 1.406 & $2.10 \pm 0.18$ & 4.5 & 0.85 & 224 & {$[-30 \div-18]$} & $234_{-41}^{+41}$ & 11.14 & $18.2 \pm 0.1$ \\
\hline $\mathrm{S} 2 \mathrm{~F} 1-443$ & 03:06:31.76 & $+00: 01: 13.4$ & 1.910 & $3.36 \pm 0.15$ & 1.9 & 0.79 & 352 & {$[-21 \div 8]$} & $357_{-64}^{+40}$ & 11.59 & $18.4 \pm 0.1$ \\
\hline
\end{tabular}

Notes. Column 1: id number; Col. 2: RA; Col. 3: Dec; Col. 4: redshift; Col. 5: circularized effective radius in kpc; Col. 6: Sersic index; Col. 7 : axis ratio; Col. 8: velocity dispersion as measured within the 1" aperture slit; Col. 9: the range in which varies the offset factor $\sigma_{\text {off }}$ to be applied to $\sigma$ according to the simulation of Appendix B; Col. 10: velocity dispersion as measured within the effective radius; Col. 11: stellar mass derived with BC03 code and Chabrier IMF; Col. 12: Vega magnitude in $K$-band (Saracco et al. 2009).

Saglia et al. sample, with that by Zahid et al. (2015), in which passive galaxies are selected on COSMOS field using a NUVrJ colour-colour cut. Using the coordinates of the galaxies available at the website ${ }^{4}$ of the author, we visually checked their morphology on HST/ACS images, finding that all but one passive galaxy are ellipticals.

For the high- $z$ sample, we collected all of the high- $z$ ETGs at $1.2<z<1.6$ with $\Sigma>2500 M_{\odot} \mathrm{pc}^{-2}, M_{\star}>10^{11} M_{\odot}$, available kinematics (i.e. velocity dispersion) and effective radius derived in the $F 160 \mathrm{~W}$ band ( 11 objects). We performed the morphological classification on the basis of a visual inspection of the galaxies carried out on the HST images in the F160W filter. Among the 11 galaxies of the total high- $z$ sample, seven galaxies were taken from the literature (see below) and, for the remaining four (plus one at $z=1.9$, hereafter "our sample of high- $z$ ETGs"), we present the unpublished VLT-FORS2 spectra together with their velocity dispersion measurements (Sect. 3.2). The seven galaxies from literature include as follow:

- Four ETGs taken from Bezanson et al. (2013). From the eight galaxies of their sample we removed the galaxy U53937 bacause it is less massive than $10^{11} M_{\odot}$, U55531 since its image shows that is not an elliptical, and C13412 and C22260, which have $\Sigma<2500 M_{\odot} \mathrm{pc}^{-2}$.

- Two ETGs taken from Belli et al. (2014). From the 56 quiescent galaxies of their sample, we excluded 23 passive galaxies that have $z<1.2$; of the remaining 33, 24 were rejected because they had $M_{\star}<10^{11} M_{\odot}$, three were not ETGs (4906, 34609 and 34265), and four (1244914, 2823, 34879, 7310) had $\Sigma<2500 M_{\odot} \mathrm{pc}^{-2}$.

\footnotetext{
4 https://www.cfa.harvard.edu/ hzahid/Data_files/ ApJ97868_table1.ascii
}

- One ETG taken from Longhetti et al. (2014). This galaxy belongs to MUNICS sample of six ETGs described in Sect. 2.1 and is used to estimate the number density.

The sample is summarized in Table 4. We do not include the three ETGs out of 17 galaxies presented in Newman et al. (2010) (ID GN3, GN4 ${ }^{b}, \mathrm{GN}^{b}$ ) with available $\sigma$, redshift $z>1.26$ and $M_{\star}>10^{11} M_{\odot}$, since their effective radii were derived fitting a de Vaucouleur and not a Sersic profile.

\subsection{Our sample of high-z ETGs: spectroscopic observations and velocity dispersion measurements}

We selected the remaining four (plus one at $z=1.91$ ) ETGs of the total high- $z$ sample (Fig. 3, HST-NIC2/F160W images from Longhetti et al. 2007) among the seven ultramassive dense ETGs spectroscopically identified at $z \sim 1.5$ in the field S2F1 of the MUNICS survey (Drory et al. 2001); thus, they belong to the MUNICS sample we used to constrain the number density of ultramassive dense ETGs at $z \sim 1.4$ (see Sect. 2.1). Their surface brightness profiles are derived by Longhetti et al. (2007) fitting the HST-NIC2/F160W images with a 2D-psf convoluted Sersic law with the software GALFIT (Peng et al. 2002). In Table 5 we report the effective radii of the best-fitted Sersic profile for the five ETGs. The circularized $R_{\mathrm{e}}[\mathrm{kpc}]$ are slightly different from those published in Longhetti et al. (2007) because of the more accurate estimates of the redshift that we derived from the higher resolution VLT-FORS2 spectra. We re-estimated stellar masses to take the updated value of redshift and the now available nearIR photometry in the WISE RSR-W1 filter $\left(\lambda_{\text {eff }} \simeq 3.4 \mu \mathrm{m}\right)$ into account. We considered a grid of composite stellar population templates derived from Bruzual \& Charlot (2003) with 

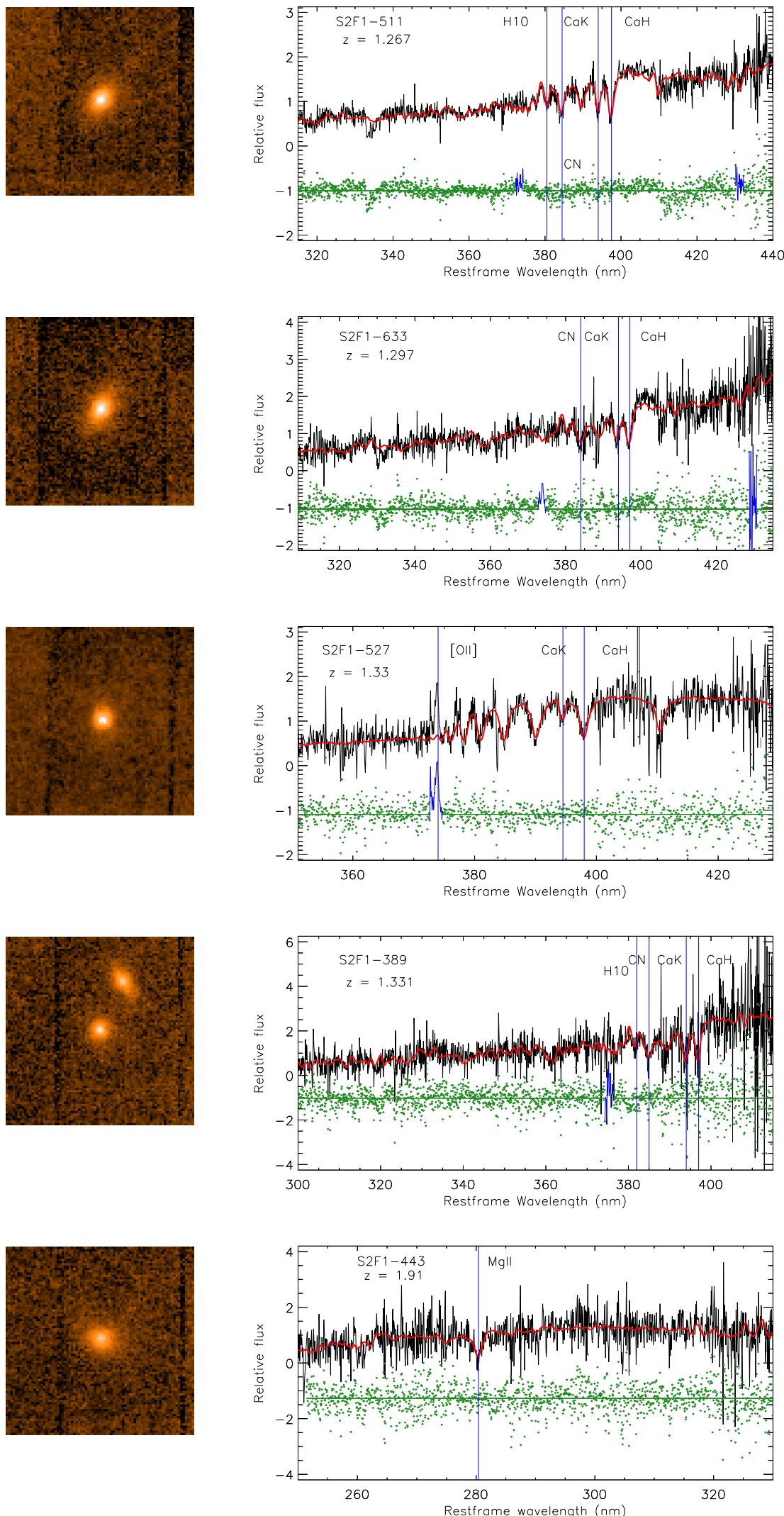

Fig. 3. Left column: HST-NIC2/F160W images of the five ETGs of our high- $z$ sample for which we present the VLT-FORS2 spectra (each image is $6 \times 6$ arcsec). Right column: spectra, unsmoothed and not-rebinned, of our five ETGs (black lines, $8 \mathrm{~h}$ of effective integration time). Many optical absorption features (blue lines) are clearly distinguishable from the continuum. The best-fit pPXF model is plotted in red. On average, pPXF selects and combines 2-4 star templates to reproduce the observed spectrum. Residuals after best-fit model subtraction are shown with green points. In the residual tracks, blue lines are the regions automatically excluded by the software pPXF as strongly affected by sky emission lines. 
solar metallicity $Z_{\odot}$, exponentially declining star formation history $(\propto \exp (-\mathrm{t} / \tau))$ with star formation timescale $\tau$ in the range 0.1-0.6 Gyr, and the Chabrier IMF (Chabrier 2003). The effect of dust extinction has been modelled following the Calzetti law (Calzetti et al. 2000) with $\mathrm{A}_{V}$ in the range [0.0-2.0]. The stellar masses $M_{\star}$ associated with the best-fit model are reported in Table 5. For the estimates of the number density derived in Sect. 2 we referred to these updated values of $R_{\mathrm{e}}$ and $M_{\star}$.

Spectroscopic observations of our five ETGs were performed with the ESO VLT-FORS2 spectrograph in MXU mode during four runs in 2010 and 2011. The OG590 filter and the GRIS-600z grism were adopted to cover the wavelength range $0.6 \mu \mathrm{m}<\lambda<$ $1.0 \mu \mathrm{m}$ with a sampling of $1.6 \AA$ per pixel. With a $1^{\prime \prime}$ slit the resulting spectral resolution is $R \simeq 1400$, corresponding to a $F W H M \simeq 6.5 \AA$ at $9000 \AA$. The total effective integration time on target galaxies is $\sim 8 \mathrm{~h}$, which results in signal-to-noise $(\mathrm{S} / \mathrm{N})$ per pixel $\sim[8-10]$ depending on the galaxy. Standard reduction was performed with IRAF tasks. In each frame the sky was removed by subtracting from each frame the following one in the dithering observing sequence. The sky-subtracted frames were aligned and co-added. The final stacked spectra were corrected for the response sensitivity function derived from the spectrum of the standard stars (FFEIGE110, GD71) observed in each of the four runs. Finally, we applied a further correction to the relative flux calibration related to the x-position of the slit on mask (Lonoce et al. 2014). In Fig. 3 the black lines show the 8-h, one dimensional spectra of our galaxies, which are not rebinned and unsmoothed.

For the velocity dispersion $(\sigma)$ measurements, we adopted the Penalized Pixel-Fitting software (Cappellari \& Emsellem 2004, pPXF), which derive the $\sigma$ by fitting the continuum and absorption features of the spectrum. We derived the velocity dispersions from the unbinned spectra, weighting each pixel for a quantity proportional to its $\mathrm{S} / \mathrm{N}$ and adopting as templates a set of 33 synthetic stars selected from the high-resolution $(R \simeq 20000)$ synthetic spectral library by Munari et al. (2005). The stars of the subsample have temperature $3500 \mathrm{~K}<T<10000 \mathrm{~K}$, $0<\log g<5$, solar abundance and metallicity and they are the same as adopted by Cappellari et al. (2009) to derive the velocity dispersions of two ETGs at $z \sim 1.4$. A detailed description of the velocity dispersion measurements are provided in Appendix $\mathrm{D}$, together with extensive set of simulations, aimed at testing the stability and accuracy of the measures, and their errors. In Table 5 we report the value of the velocity dispersion $(\sigma)$ corrected for instrumental dispersion $\left(\sim 110 \mathrm{~km} \mathrm{~s}^{-1}\right)$ and their errors (see Appendix D). Since data refer to $1^{\prime \prime}$ slit, the measured $\sigma$ have to be considered within a radius of 0.5 arcsec. In Table 1 we also report $\sigma_{\mathrm{e}}$, i.e. the velocity dispersion within the effective radius. We scaled the measured $\sigma$ to $\sigma_{\mathrm{e}}$, following Cappellari et al (2006).

\subsection{The evolution of structural and dynamical parameters of ultramassive dense ETGs: results}

In the galaxy selection, we paid particular attention to the filter in which structural parameters were derived. The effective radii were derived in the $F 160 \mathrm{~W}$ band for all of the galaxies at $1.2<z<1.6$, in the $F 814 W$ band for the sample at intermediate redshift range, and in the $r$-band for the local SDSS galaxies. This allowed us to sample approximately the same rest-frame band through all of the redshift range we probed.

Figure 4 shows the distribution of ultramassive dense ETGs at $0 \lesssim z<1.6$ in the plane defined by effective radius and stellar

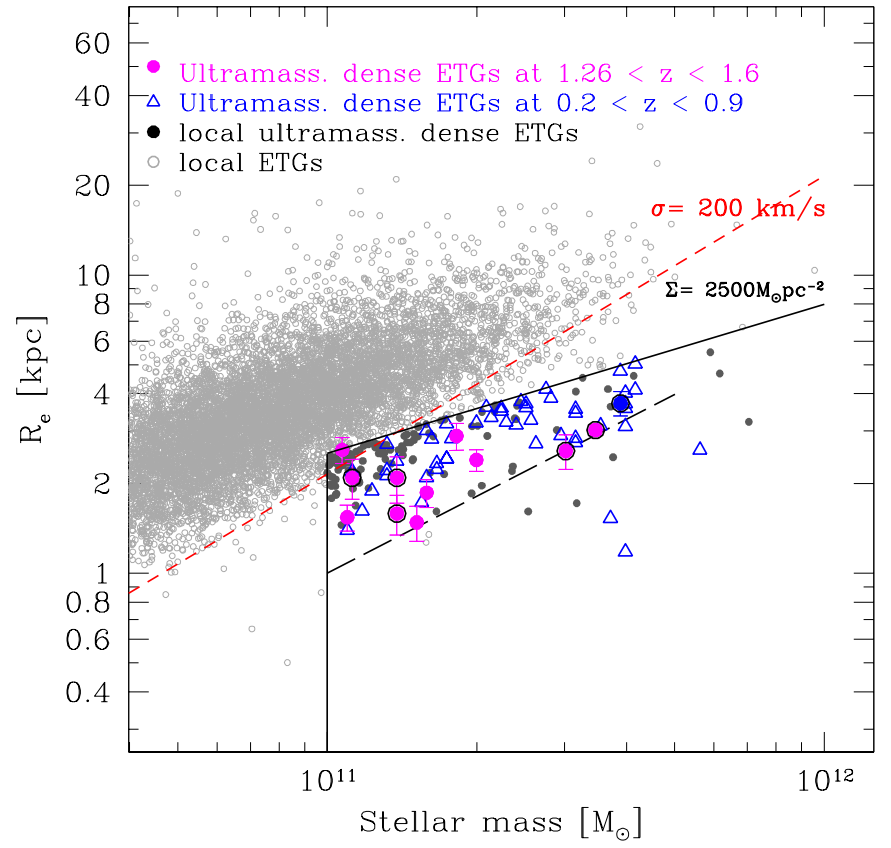

Fig. 4. Distribution of the ultramassive dense ETGs from $z \sim 1.6$ to $z \sim 0$ in the $R_{\mathrm{e}}-M_{\star}$ plane. Ultramassive dense ETGs at $1.2<z<1.6$ are represented with magenta filled circles. The ETGs from MUNICS sample with available velocity dispersion (five presented here and one in Longhetti et al. 2014) are indicated with black countours. The ETG of MUNICS sample at redshift 1.91 (hence not included in the analysis of Sect. 3.3) is indicated with a blue point. Ultramassive dense ETGs at $0.3<z<0.9$ and in the local Universe are indicated with blue open triangles and dark grey points, respectively. Solid black lines define the cuts in $M_{\star}$ and $\Sigma$ of our samples. The small open circles show local ETGs from Thomas et al. (2010) in the redshift range $0.063<z<0.1$. The red line indicates a line of constant $\sigma$ derived as $R_{\mathrm{e}}=\sqrt{G M_{\star} / 5 \sigma}$, i.e. assuming zero dark matter. The long-dashed line indicates the lower boundary of the size-mass relation as found by Belli et al. (2014) and van der Wel et al. (2014).

mass. The six spheroids belonging to MUNICS sample (the five presented in Sect. 3.2 plus one by Longhetti et al. 2014) are highlighted with black contours and we highlighted the ultramassive ETG of our high- $z$ sample at $z=1.91$ with a blue point. This galaxy is not included in the comparison with local ETGs.

Given the request of available velocity dispersion, the sample we collected is clearly not complete and does not homogeneously cover the region of the size-mass plane occupied by dense galaxies. We checked, however, that the request of available velocity dispersion does not produce a bias against the most compact objects, i.e. that we are not missing the smallest ETGs at fixed stellar mass. In Fig. 5 the long-dashed black line is the lower boundary of the size-mass relation found by Belli et al. (2014) and van der Wel et al. (2014); these are good agreement with each other. Although our sample is not complete, it is not biased against dense ETGs, as expected since velocity dispersion measurements are more successful in dense systems.

In the left and right panels of Fig. 5 we plot the velocity dispersion of ultramassive dense ETGs as a function of stellar mass and effective radius, respectively. The first thing that we may note is that all of the ultramassive dense ETGs at high- $z$ have an ultramassive dense counterpart in the local Universe with comparable structural and dynamical properties. Also the two most massive $\left(M_{\star} \gtrsim 3 \times 10^{11} M_{\odot}\right)$ and densest $\left(\Sigma>5000 M_{\odot} \mathrm{pc}^{-2}\right)$ high- $z$ ETGs, whose parameters are offset with respect to the other high- $z$ dense ETGs, have counterparts. Their counterparts 


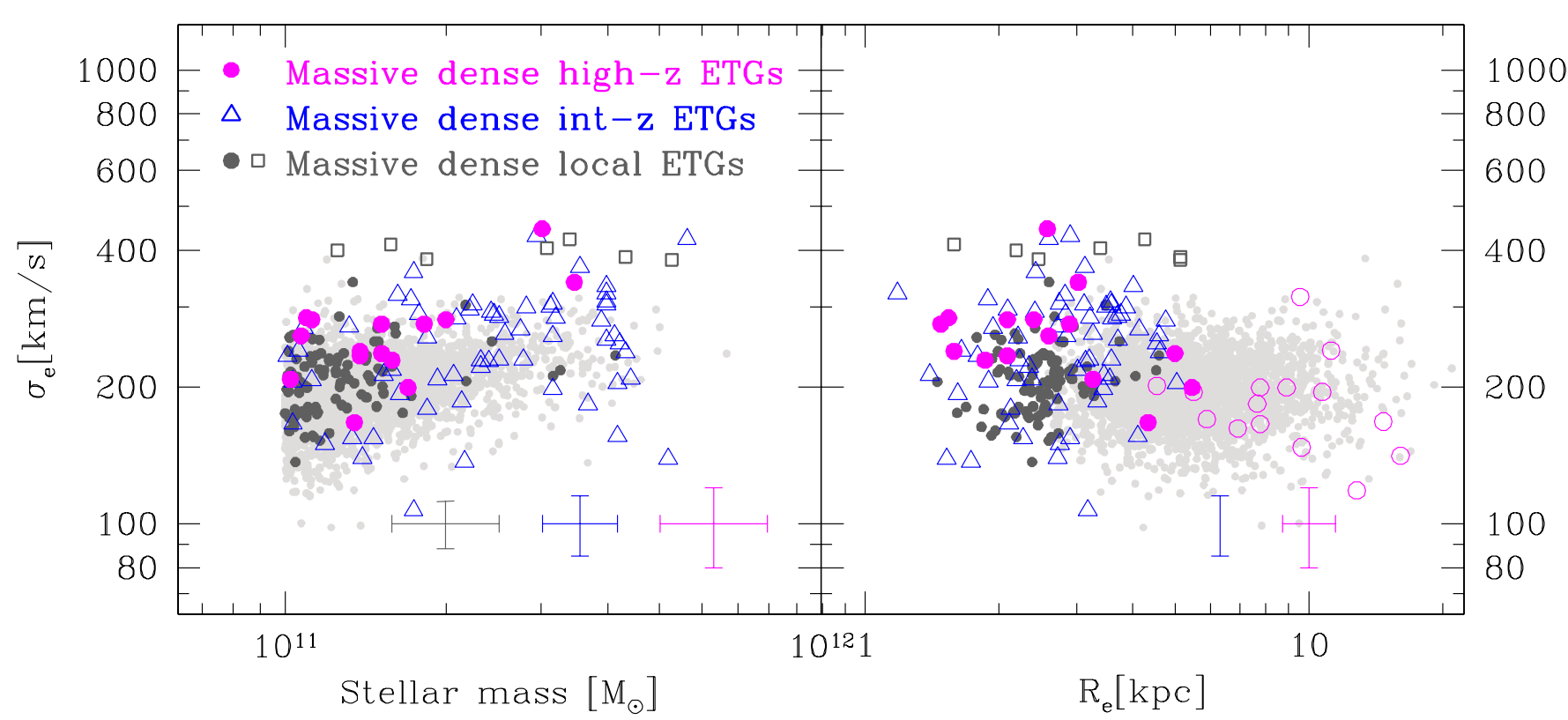

Fig. 5. Comparison between the structural and dynamical properties of ultramassive dense ETGs over the last $\sim 10$ Gyr. Magenta points indicate dense ETGs at $1.2 \lessgtr z<1.6$, open blue triangles indicate dense ETGs at $0.3 \lessgtr z \lesssim 0.9$, and local ETGs are shown with dark grey small dots. Light grey points are the whole sample of local ETGs with $M_{\star}>10^{11} M_{\odot}$, thus with no cut in mean stellar mass density. Grey open squares indicate the local ultramassive dense galaxies selected from the sample of local galaxies with velocity dispersion $>350 \mathrm{~km} \mathrm{~s}^{-1}$ by Bernardi et al. (2008), while open magenta circles show high- $z$ ETGs evolved in size a factor $\sim 3$, as predicted by the relation $\left\langle R_{\mathrm{e}}\right\rangle \propto(1+z)^{-1.5}$ and in $\sigma$ according to the merger scenario by (Hopkins et al. 2009). In each panel, average error bars are reported. They are colour coded according to the sample they refer. For what concerns the high- $z$ sample, we have found that on average $\delta_{R_{\mathrm{e}}} / R_{\mathrm{e}} \sim 11 \%, \delta_{\sigma_{\mathrm{e}}} / \sigma_{\mathrm{e}} \sim 10 \%$ (see Tables 3 and 4 ), while we have assumed a typical uncertainty of $20 \%$ on $M_{\star}$. At intermediate redshift, $\delta_{R_{\mathrm{e}}} / R_{\mathrm{e}} \sim 10 \%, \delta_{\sigma_{\mathrm{e}}} / \sigma_{\mathrm{e}} \sim 7 \%$, and $\delta_{M_{\star}}=0.10$ dex (see Saglia et al. 2010). In local Universe, $\delta_{M_{\star}}=0.07 \mathrm{dex}$ (see Salim et al. 2007), $\delta_{\sigma_{\mathrm{e}}} / \sigma_{\mathrm{e}} \sim 10 \%$, and we assume $\delta_{R_{\mathrm{e}}} / R_{\mathrm{e}} \sim 15 \%$. For the error on local $R_{\mathrm{e}}$ we refer to La Barbera et al. (2010) which derive the structural parameters of SDSS galaxies on SDSS images using 2DPHOT. In fact, the errors on parameters are expected to be mainly driven by the characteristics of the images, and not by the software and/or procedure used.

can be found among the local ultramassive dense galaxies belonging to the sample of SDSS galaxies with $\sigma>350 \mathrm{~km} \mathrm{~s}^{-1}$ selected by Bernardi et al. (2008) (Fig. 5 open squares). Hence, Fig. 6 shows that in local Universe there are ultramassive dense ETGs with effective radii and velocity dispersions comparable to those of equally massive and dense ETGs at $\langle z\rangle=1.4$. At the same time, Fig. 5 shows that there are some local galaxies with $\sigma_{\mathrm{e}}<200 \mathrm{~km} \mathrm{~s}^{-1}$ without high- $z$ counterparts. These ETGs populate the region in Fig. 4 immediately below the line at $\Sigma=2500 M_{\odot} \mathrm{pc}^{-2}$ with $10^{11}<M_{\star}<2 \times 10^{11}$, where indeed no high $-z$ galaxies fall. Actually, the sparse sampling of the high- $z$ sample and its incompleteness, prevent us from assesing whether the lack of low- $\sigma$ ultramassive dense ETGs at high- $z$ is real or not.

As an exercise, we show, with open circles in Fig. 5, the displacement that high- $z$ dense ETGs would show in the space $\left(R_{\mathrm{e}}\right.$, $\left.M_{\star}, \sigma_{\mathrm{e}}\right)$ under the hypothesis that they evolve in size according to the relation $(1+z)^{-1.5}$ (i.e. a factor $\sim 3$ from $z \sim 1.4$ to $z=0$, as found e.g. by Cassata et al. 2013; Cimatti et al. 2012; van der Wel et al. 2014), and in $\sigma$ according to the merging scenario proposed by Hopkins et al. (2009). Thus, if all or most of the high- $z$ ultramassive dense ETGs evolve in size (and $\sigma_{\mathrm{e}}$ ), they have to be replaced by other ultramassive dense ETGs along the time to restore the population seen in the local Universe.

\section{Discussion and conclusions}

We have investigated the mass accretion history of ultramassive dense $\left(M_{\star}>10^{11} M_{\odot}, \Sigma>2500 M_{\odot} \mathrm{pc}^{-2}\right)$ ETGs, elliptical and spheroidal galaxies selected on the basis of visual selected, over the last $\sim 9$ Gyr. We addressed this topic by tracing the evolution of their comoving number density and comparing the structural (effective radius $R_{\mathrm{e}}$ and stellar mass $M_{\star}$ ) and dynamical (velocity dispersion $\sigma_{\mathrm{e}}$ ) parameters of ultramassive dense ETGs from redshift $\sim 1.6$ to redshift $\sim 0$.

We took advantage of the MUNICS and GOODS-South surveys to constrain the comoving number density $\rho$ of ultramassive dense ETGs since at $1<z<1.6$, while we used the COSMOS spectroscopic survey to cover the redshift range from $z=1.0$ to $z=0.2$. For the number density of ultramassive dense ETGs in the local Universe, we referred to the sample of local ETGs selected from the SDSS DR4 by Thomas et al. (2010). We found that the comoving number density follows the relation $\rho \propto(1+z)^{0.3 \pm 0.8}$, i.e. it decreases at most by $25 \%$ since $z=1.6$.

Our results are in good agreement with the other findings presented in literature (Carollo et al. 2013; Damjanov et al. 2015; Cassata et al. 2013; Valentinuzzi et al. 2010; Bezanson et al. 2013), with the only two exceptions concerning the estimates provided by Taylor et al. (2010a) at $z \sim 0$, and by van der Wel et al. (2014) at $0.2<z<1.5$; in both cases our number densities are greater than those claimed by the authors. In the first case, we have shown that the main source of the observed discrepancy is in the different definition of $R_{\mathrm{e}}$ used; semi-major axes $\mathrm{SM}_{\mathrm{e}}$ that were obtained fitting a de Vaucouleur profile in the Taylor investigation versus circularized $R_{\mathrm{e}}$ that was obtained fitting a Sersic profile in our analysis. In the second case, we argue that the main reason for the drastic ( $\sim$ one order of magnitude) drop observed in the number density by van der Wel et al. (2014) can be ascribed to the procedure they adopted to derive 
the effective radius at $5000 \AA$ and the usage of semi-major axis $\mathrm{SM}_{\mathrm{e}}$.

We then investigated whether the ultramassive dense ETGs observed at high- $z$ have a local counterpart, i.e. whether they are similar both in their structure $\left(R_{\mathrm{e}}\right.$ and $\left.M_{\star}\right)$ and kinematics $\left(\sigma_{\mathrm{e}}\right)$ to the low-z ETGs. To this aim, we collected a homogeneous sample of ultramassive dense ETGs at $1.2<z<1.6$ with available $R_{\mathrm{e}}, M_{\star}$, and $\sigma_{\mathrm{e}}$. We only included in our final catalogue the galaxies with clear elliptical morphology, effective radius derived in the $F 160 \mathrm{~W}$ band fitting a Sersic profile, stellar masses estimated with BC03 models, and a Chabrier IMF. The request of available velocity dispersion restricts the sample to 11 ETGs. For four ETGs out of 11 (plus one at $z=1.91$ ), we presented new unpublished VLT-FORS2 spectra and the measure of their velocity dispersions. For the intermediate redshift range $(0.2<z<0.9)$, we refer to the sample of ETGs by Saglia et al. (2010) and to the sample of passive galaxies by Zahid et al. (2015), while as local reference we adopted the complete sample of local $(0.063<z<0.1)$ ETGs selected from the SDSS DR4 by Thomas et al. (2010). The comparison in the plane $\left[R_{\mathrm{e}}, M_{\star}, \sigma_{\mathrm{e}}\right]$ shows that all of the ultramassive dense ETGs at $z=1.4$ have a local counterpart with a similar velocity dispersion, stellar mass, and effective radius.

The two pieces of evidences above point towards two simple scenarios. In the last $9-10$ Gyr, the vast majority ( $~ 270 \%$, according to the number density) of ultramassive dense ETGs passively age, leaving the bulk of the stellar mass, kinematics, and structure almost unchanged. In this case, the observed size growth of ultramassive ETGs has to be ascribed mostly to newborn larger ETGs. The other possibility is that a significant portion of high$z$ ultramassive dense ETGs migrate, through inside-out accretion, towards less dense systems, thereby sustaining the mean size growth of spheroidal galaxies. Concurrently, the emergence of new ultramassive dense ETGs at lower redshift maintains the number density at almost constant. We are not in the position to discriminate among the two possibilities, but some considerations deserve to be discussed.

A conventional approach to distinguish the real descendants from the newly formed spheroids (i.e. to discriminate among the two above scenarios) is to compare high redshift galaxies with old (on the basis of their luminosity weighted age LWA) low- $z$ galaxies. However, this method does not take into account the evidence that despite the old global age, spheroidal galaxies often host very young stars (e.g. La Barbera et al. 2012). Although this population of young stars has a negligible weight in terms of total stellar mass (e.g. Tantalo \& Chiosi 2004), these young stars can significantly lower the LWA. The direct consequence of this is that to select local descendants through a cut in LWA can exclude a portion of spheroidal galaxies already in place at $z \sim 1.4$. As a further complication, most of the methods used to infer galaxies age are limited by the age-metallicity degeneracy and by the possible presence of the dust.

Considering these aspects, we decided to investigate the distribution of local ultramassive dense ETGs in the $D_{n}(4000)-$ $\mathrm{H}_{\delta}$ plane. Actually, the $D_{n}(4000)$ index progressively increases with the age of the stellar population, while the strong absorption $\mathrm{H}_{\delta}$ line arises in galaxies that have experienced a burst of star formation in the past 1-2 Gyr. Although both indices are metallicity sensitive, their combination is not so and, moreover, is largely insensitive to the dust-attenuation effects. In Fig. 6 we reported the $D_{n}(4000)$ and $\mathrm{H}_{\delta}$ values for our sample of $124 \mathrm{ul}-$ tramassive dense local ETGs. For the spectral indices we referred to the database provided by Kauffmann et al. (2003) for SDSS galaxies. The figure shows that just $\sim 70 \%(60 \%)$ of local

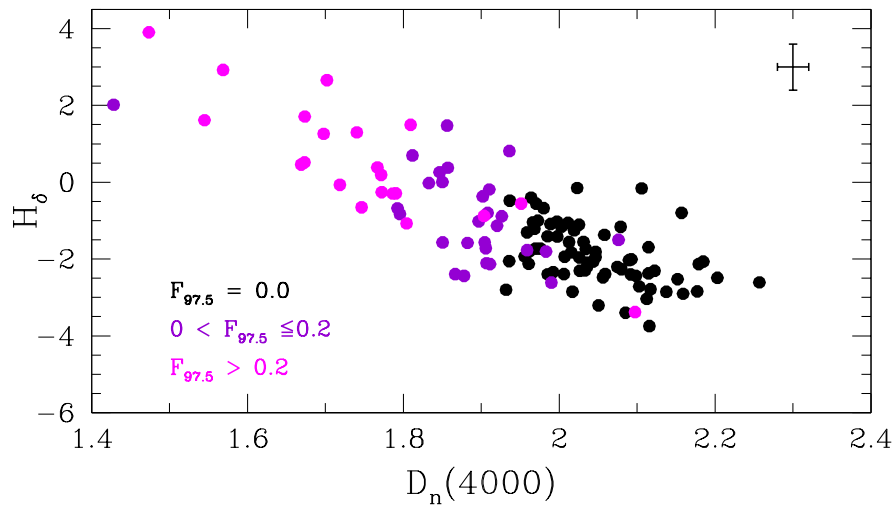

Fig. 6. The $\mathrm{H}_{\delta}$ of ultramassive dense local ETGs versus their $D_{n}(4000)$ colour coded on the basis of the 97.5 percentile value of $F$, where $F$ is the fraction of stellar mass formed in the last $2 \mathrm{Gyr}$.

ultramassive dense ETGs have $D_{n}(4000) \gtrsim 1.9(1.95)$ (thus age $\gtrsim 9 \mathrm{Gyr}$ ), therefore, on the basis of their age, only two out of three local ETGs should be considered descendants of high- $z$ compact ETGs. For each galaxy, however, Kauffmann et al. (2003) best fitted the observed values of $D_{n}(4000)$ and $\mathrm{H}_{\delta}$ with a grid of composite stellar population (CSP) models, which both had typical declining and bursty star formation history. From the best-fit model, they derived the fraction $F$ of stellar mass formed in the last 2 Gyr and provided both its median value and the 2.5 and 97.5 percentile of its likelihood distribution. Actually, all of the galaxies in Fig. 6 have a median value of $F=0$. However, in Fig. 6 we colour coded the galaxies on the basis of the 97.5 percentile value of $F\left(F_{97.5}\right)$. This is because beyond the exact value of $F$, which can be susceptible to the grid of the input models and prior adoption in the fit, we are interested in investigating whether there is a non-null probability to have a secondary (minor) event of star formation, which can drift the age estimate towards a lower value. Interestingly, in the vast majority of the galaxies with $D_{n}(4000) \gtrsim 1.9$ (and less than $10 \%$ for galaxies with $\left.D_{n}(4000) \gtrsim 1.95\right), F_{97.5}=0$. For the remaining galaxies of the sample with $D_{n}(4000)<1.9$, their $F_{97.5}$ value is $>0$, thus there is a non-null probability that they experienced a burst of star formation in the last 2 Gyr. In this context, detailed studies on spectral features of passive galaxies (Lonoce et al. 2014) have shown that fitting the $D_{n}(4000)$ with a single CSP model with a declining star formation history returns age values that can be significantly (even $\gtrsim 50 \%$ ) lower than the values obtained with a SED fitting or obtained assuming a composite model made by an old component forming the bulk of the stellar mass, and a young stellar population contributing just few $\%$ to the total stellar mass amount. If this is the case, the galaxies with $D_{n}(4000)<1.9$ could be still considered descendant of high- $z$ ETGs that experienced secondary and minor events of star formation at later epochs (see also Thomas et al. 2010). These events can have a drastic impact on the estimate of galaxy age, but do not significantly alter the stellar mass. Actually, Fig. 6 shows that the probability of a galaxy increasing its stellar mass by more than $20 \%$ in secondary events of star formation is extremely low.

This analysis is not intended to be quantitative, but to show that the treatment of the progenitor bias is very complex and that the mere selection of local descendants on the basis of the galaxy age that is obtained through the photometry fit or even through the more robust $D_{n}(4000)$, can provide at most a lower limit to the true number of local descendants. A more detailed analysis of the whole ensemble of the spectral features that are age and 
"star-formation history" sensitive (e.g. $\left.\mathrm{H}_{\delta} ; \mathrm{H}+\mathrm{K}, D_{n}(4000)\right)$ is mandatory both in local and at intermediate redshift range, to track the real mass assembly history of ETGs.

Although not conclusive, the analysis of spectral features of local ETGs have shown that there is a non-null probability that all of the local dense ETGs are high- $z$ descendants. Moreover, in recent years, studies on the properties (number densities, masses, sizes, SFRs) of submillimeter galaxies (SMGs) have strengthened the possible evolutionary connection between these objects and dense high- $z$ elliptical, in the direction that the SMGs are the progenitors of the dense ETGs (e.g. Barro et al. 2013, 2014; Toft et al. 2014). Nonetheless, these highly star-forming galaxies are extremely rare at $z<1$. If galaxies with, for example, $D_{n}(4000)<1.9$ are genuinely young ultradense systems (and not old galaxies that have experienced recent star-formation events), we should find a way to model their formation. The lack of progenitors for young dense ETGs, strengthens the hypothesis that local compact massive galaxies are descendants of high redshift galaxies.

Thus, more than one piece of observational evidence would point towards the first of the two proposed scenarios according to which most $(\gtrsim 75 \%)$ of the ultramassive dense ETGs observed at $z \sim 1.4$ have already completed their assembly. Although a residual event of star formation may occur, these cannot significantly modify neither the mass, the velocity dispersion, nor the typical dimension of the ultramassive dense galaxies already formed at $z \sim 1.4$. As far as the remaining $25 \%$ of dense ETGs possibly missing in the local Universe, stochastic merger events could have transformed them into non-dense systems $\left(\Sigma \leq 2500 M_{\odot} \mathrm{pc}^{-2}\right)$, contributing to the increase of the mean size and the number density of the whole population of ultramassive ETGs at $z<1.5$. In fact, de la Rosa et al. (2016) have found that a portion of presentday massive galaxies host in the centre a compact core with the same structural and dynamical properties as high- $z$ compact galaxies. Numerically, the contribution of these size-evolved ultramassive dense ETGs to the total number density of local ultramassive ETGs is $\sim 2 \times 10^{-5} \mathrm{gal} / \mathrm{Mpc} 3$ (see Fig. 2). Actually, the number density of passive $M_{\star}>10^{11} M_{\odot}$ galaxies increases from $\sim 10^{-4}$ to $5 \times 10^{-4} \mathrm{gal} / \mathrm{Mpc}^{-4}$ from redshift 1.5 to $z \sim 0$ (Ilbert et al. 2010; Pozzetti et al. 2010; Brammer et al. 2011; Moustakas et al. 2013; Muzzin et al. 2013). Therefore, if the fraction of newly formed ultramassive dense ETGs is negligible, the eventual contribution of the size-evolved ultramassive dense ETGs to the mean size growth in terms of number density is $\lesssim 5 \%$.

Acknowledgements. We warmly thank the anonymous referee for his/her comments and suggestions which, in our opinion, have really improved the whole manuscript. This work has received financial support from PrinINAF 1.05.09.01.05 and is based on observations made with ESO Very Large Telescope under programme ID 085.A-0135A.

\section{References}

Adelman-McCarthy, J. K., Agüeros, M. A., Allam, S. S., et al. 2006, ApJS, 162, 38

Barro, G., Faber, S. M., Pérez-González, P. G., et al. 2013, ApJ, 765, 104

Barro, G., Trump, J. R., Koo, D. C., et al. 2014, ApJ, 795, 145

Belli, S., Newman, A. B., \& Ellis, R. S. 2014, ApJ, 783, 117

Bernardi, M. 2009, MNRAS, 395, 1491

Bernardi, M., Hyde, J. B., Fritz, A., et al. 2008, MNRAS, 391, 1191

Bezanson, R., van Dokkum, P. G., Tal, T., et al. 2009, ApJ, 697, 1290

Bezanson, R., van Dokkum, P., \& Franx, M. 2012, ApJ, 760, 62

Bezanson, R., van Dokkum, P., van de Sande, J., Franx, M., \& Kriek, M. 2013, ApJ, 764, L8
Blanton, M. R., \& Roweis, S. 2007, AJ, 133, 734

Blanton, M. R., Schlegel, D. J., Strauss, M. A., et al. 2005, AJ, 129, 2562

Brammer, G. B., Whitaker, K. E., van Dokkum, P. G., et al. 2011, ApJ, 739, 24

Bruce, V. A., Dunlop, J. S., Cirasuolo, M., et al. 2012, MNRAS, 427, 1666

Bruzual, G., \& Charlot, S. 2003, MNRAS, 344, 1000

Buitrago, F., Trujillo, I., Conselice, C. J., et al. 2008, ApJ, 687, L61

Buitrago, F., Trujillo, I., Conselice, C. J., \& Häußler, B. 2013, MNRAS, 428, 1460

Calzetti, D., Armus, L., Bohlin, R. C., et al. 2000, ApJ, 533, 682

Cappellari, M., \& Emsellem, E. 2004, PASP, 116, 138

Cappellari, M., Bacon, R., Bureau, M., et al. 2006, MNRAS, 366, 1126

Cappellari, M., di Serego Alighieri, S., Cimatti, A., et al. 2009, ApJ, 704, L34

Cappellari, M., Emsellem, E., Krajnović, D., et al. 2011, MNRAS, 413, 813

Carollo, C. M., Bschorr, T. J., Renzini, A., et al. 2013, ApJ, 773, 112

Carter, D., Goudfrooij, P., Mobasher, B., et al. 2008, ApJS, 176, 424

Cassata, P., Giavalisco, M., Guo, Y., et al. 2011, ApJ, 743, 96

Cassata, P., Giavalisco, M., Williams, C. C., et al. 2013, ApJ, 775, 106

Chabrier, G. 2003, PASP, 115, 763

Cimatti, A., Cassata, P., Pozzetti, L., et al. 2008, A\&A, 482, 21

Cimatti, A., Nipoti, C., \& Cassata, P. 2012, MNRAS, 422, L62

Daddi, E., Renzini, A., Pirzkal, N., et al. 2005, ApJ, 626, 680

Damjanov, I., McCarthy, P. J., Abraham, R. G., et al. 2009, ApJ, 695, 101 Damjanov, I., Abraham, R. G., Glazebrook, K., et al. 2011, ApJ, 739, L44

Damjanov, I., Hwang, H. S., Geller, M. J., \& Chilingarian, I. 2014, ApJ, 793, 39

Damjanov, I., Geller, M. J., Zahid, H. J., \& Hwang, H. S. 2015, ApJ, 806, 158

Davies, L. J. M., Driver, S. P., Robotham, A. S. G., et al. 2015, MNRAS, 447, 1014

de la Rosa, I. G., La Barbera, F., Ferreras, I., et al. 2016, MNRAS, 457, 1916 Drory, N., Feulner, G., Bender, R., et al. 2001, MNRAS, 325, 550

Franx, M., van Dokkum, P. G., Schreiber, N. M. F., et al. 2008, ApJ, 688, 770

Gargiulo, A., Saracco, P., Longhetti, M., La Barbera, F., \& Tamburri, S. 2012, MNRAS, 425, 2698

Giavalisco, M., Ferguson, H. C., Koekemoer, A. M., et al. 2004, ApJ, 600, L93

Grogin, N. A., Kocevski, D. D., Faber, S. M., et al. 2011, ApJS, 197, 35

Guo, Y., McIntosh, D. H., Mo, H. J., et al. 2009, MNRAS, 398, 1129

Guo, Y., Giavalisco, M., Cassata, P., et al. 2011, ApJ, 735, 18

Hopkins, P. F., Bundy, K., Murray, N., et al. 2009, MNRAS, 398, 898

Hoyos, C., den Brok, M., Verdoes Kleijn, G., et al. 2011, MNRAS, 411, 2439

Huertas-Company, M., Mei, S., Shankar, F., et al. 2013, MNRAS, 428, 1715

Ilbert, O., Salvato, M., Le Floc'h, E., et al. 2010, ApJ, 709, 644

Ilbert, O., McCracken, H. J., Le Fèvre, O., et al. 2013, A\&A, 556, A55

Jorgensen, I., \& Chiboucas, K. 2013, AJ, 145, 77

Jorgensen, I., Chiboucas, K., Toft, S., et al. 2014, AJ, 148, 117

Kauffmann, G., Heckman, T. M., White, S. D. M., et al. 2003, MNRAS, 341, 33

Keating, S. K., Abraham, R. G., Schiavon, R., et al. 2015, ApJ, 798, 26

Koekemoer, A. M., Aussel, H., Calzetti, D., et al. 2007, ApJS, 172, 196

Koekemoer, A. M., Faber, S. M., Ferguson, H. C., et al. 2011, ApJS, 197, 36

Kormendy, J. 1982, ApJ, 257, 75

Kormendy, J., \& Illingworth, G. 1982, ApJ, 256, 460

Kroupa, P. 2001, MNRAS, 322, 231

La Barbera, F., de Carvalho, R. R., de La Rosa, I. G., et al. 2010, MNRAS, 408, 1313

La Barbera, F., Ferreras, I., de Carvalho, R. R., et al. 2012, MNRAS, 426, 2300

Longhetti, M., Saracco, P., Severgnini, P., et al. 2007, MNRAS, 374, 614

Longhetti, M., Saracco, P., Gargiulo, A., Tamburri, S., \& Lonoce, I. 2014, MNRAS, 439, 3962

Lonoce, I., Longhetti, M., Saracco, P., Gargiulo, A., \& Tamburri, S. 2014, MNRAS, 444, 2048

Mancini, C., Matute, I., Cimatti, A., et al. 2009, A\&A, 500, 705

Mancini, C., Daddi, E., Renzini, A., et al. 2010, MNRAS, 401, 933

Maraston, C., \& Strömbäck, G. 2011, MNRAS, 418, 2785

Marchesini, D., van Dokkum, P. G., Förster Schreiber, N. M., et al. 2009, ApJ, 701, 1765

Moresco, M., Pozzetti, L., Cimatti, A., et al. 2013, A\&A, 558, A61

Moustakas, J., Coil, A. L., Aird, J., et al. 2013, ApJ, 767, 50

Munari, U., Sordo, R., Castelli, F., \& Zwitter, T. 2005, A\&A, 442, 1127

Muzzin, A., Marchesini, D., Stefanon, M., et al. 2013, ApJ, 777, 18

Newman, A. B., Ellis, R. S., Treu, T., \& Bundy, K. 2010, ApJ, 717, L103

Newman, A. B., Ellis, R. S., Bundy, K., \& Treu, T. 2012, ApJ, 746, 162

Peng, C. Y., Ho, L. C., Impey, C. D., \& Rix, H. 2002, AJ, 124, 266

Poggianti, B. M., Calvi, R., Bindoni, D., et al. 2013a, ApJ, 762, 77

Poggianti, B. M., Moretti, A., Calvi, R., et al. 2013b, ApJ, 777, 125

Pozzetti, L., Bolzonella, M., Zucca, E., et al. 2010, A\&A, 523, A13

Saglia, R. P., Sánchez-Blázquez, P., Bender, R., et al. 2010, A\&A, 524, A6

Salim, S., Rich, R. M., Charlot, S., et al. 2007, ApJS, 173, 267

Saracco, P., Longhetti, M., Severgnini, P., et al. 2005, MNRAS, 357, L40

Saracco, P., Longhetti, M., \& Andreon, S. 2009, MNRAS, 392, 718

Saracco, P., Longhetti, M., \& Gargiulo, A. 2010, MNRAS, L115 
A. Gargiulo et al.: Number density evolution of ultra-massive dense early-type galaxies since $z=1.6$

Saracco, P., Longhetti, M., \& Gargiulo, A. 2011, MNRAS, 412, 2707

Saracco, P., Casati, A., Gargiulo, A., et al. 2014, A\&A, 567, A94

Sargent, M. T., Carollo, C. M., Lilly, S. J., et al. 2007, ApJS, 172, 434

Scarlata, C., Carollo, C. M., Lilly, S., et al. 2007, ApJS, 172, 406

Severgnini, P., Della Ceca, R., Braito, V., et al. 2005, A\&A, 431, 87

Shen, S., Mo, H. J., White, S. D. M., et al. 2003, MNRAS, 343, 978

Simard, L., Mendel, J. T., Patton, D. R., Ellison, S. L., \& McConnachie, A. W. 2011, ApJS, 196, 11

Stott, J. P., Collins, C. A., Burke, C., Hamilton-Morris, V., \& Smith, G. P. 2011, MNRAS, 414, 445

Strauss, M. A., Weinberg, D. H., Lupton, R. H., et al. 2002, AJ, 124, 1810

Szomoru, D., Franx, M., Bouwens, R. J., et al. 2011, ApJ, 735, L22

Szomoru, D., Franx, M., \& van Dokkum, P. G. 2012, ApJ, 749, 121

Tamburri, S., Saracco, P., Longhetti, M., et al. 2014, A\&A, 570, A102

Tantalo, R., \& Chiosi, C. 2004, MNRAS, 353, 405

Taylor, E. N., Franx, M., Brinchmann, J., van der Wel, A., \& van Dokkum, P. G. 2010a, ApJ, 722, 1

Taylor, E. N., Franx, M., Glazebrook, K., et al. 2010b, ApJ, 720, 723

Thomas, D., Maraston, C., Schawinski, K., Sarzi, M., \& Silk, J. 2010, MNRAS, 404, 1775
Thomas, D., Steele, O., Maraston, C., et al. 2013, MNRAS, 431, 1383

Toft, S., van Dokkum, P., Franx, M., et al. 2007, ApJ, 671, 285

Toft, S., Smolčić, V., Magnelli, B., et al. 2014, ApJ, 782, 68

Tortora, C., La Barbera, F., Napolitano, N. R., et al. 2016, MNRAS, 457, 2845

Treu, T., Stiavelli, M., Møller, P., Casertano, S., \& Bertin, G. 2001, MNRAS, 326, 221

Trujillo, I., Feulner, G., Goranova, Y., et al. 2006, MNRAS, 373, L36 Trujillo, I., Conselice, C. J., Bundy, K., et al. 2007, MNRAS, 382, 109

Trujillo, I., Cenarro, A. J., de Lorenzo-Cáceres, A., et al. 2009, ApJ, 692, L118 Valentinuzzi, T., Fritz, J., Poggianti, B. M., et al. 2010, ApJ, 712, 226 van de Sande, J., Kriek, M., Franx, M., et al. 2013, ApJ, 771, 85 van der Wel, A., Holden, B. P., Zirm, A. W., et al. 2008, ApJ, 688, 48 van der Wel, A., Rix, H.-W., Wuyts, S., et al. 2011, ApJ, 730, 38 van der Wel, A., Bell, E. F., Häussler, B., et al. 2012, ApJS, 203, 24 van der Wel, A., Franx, M., van Dokkum, P. G., et al. 2014, ApJ, 788, 28 van Dokkum, P. G., Franx, M., Kriek, M., et al. 2008, ApJ, 677, L5 Wake, D. A., van Dokkum, P. G., \& Franx, M. 2012, ApJ, 751, L44 Windhorst, R. A., Cohen, S. H., Hathi, N. P., et al. 2011, ApJS, 193, 27

Zahid, H. J., Damjanov, I., Geller, M. J., \& Chilingarian, I. 2015, ApJ, 806, 122 Zirm, A. W., van der Wel, A., Franx, M., et al. 2007, ApJ, 656, 66 


\section{Appendix A: Testing the reliability of $R_{\mathrm{e}}$ and $M_{\star}$ for local SDSS ETGs}

\section{A.1. Effective radius of local SDSS ETGs}

The effective radius for SDSS galaxies has been derived by many authors adopting different methods (e.g. a 1D fit with the NYUVAC pipeline by Blanton et al. 2005; a 2D fit with GALFIT by Simard et al. 2011 and Guo et al. 2009) and it is not straightforward to assess which analysis provides the more accurate estimate of the parameter. In fact, all of these works have estimated the $R_{\mathrm{e}}$ on SDSS images (PSF- $F W H M=1.4^{\prime \prime}$ in $r$-band, pixel size $=0.396^{\prime \prime} / \mathrm{px}$, i.e. $\sim 0.56 \mathrm{kpc} / \mathrm{px}$ at $\left.z=0.075\right)$, and thus the central region of the light profile (very sensitive to the Sersic index) of a typical ultramassive dense ETG is sampled by just 3-4 px in the redshift range we probed. Based on this evidence, to test the accuracy of the $R_{\mathrm{e}}$ we used in this work, we compared the estimate of the effective radius derived by Blanton et al. (2005) from SDSS images, with those obtained using HST optical images $\left(F W H M \simeq 0.12^{\prime \prime}\right.$, pixel size $\left.0.05^{\prime \prime} / \mathrm{px}\right)$. We used the Canadian Astronomy Data Centre ${ }^{5}$ to find ACS-HST images in the $F 775 \mathrm{~W} / \mathrm{F} 814 \mathrm{~W} / \mathrm{F} 850 \mathrm{LP} /$ filter for the 124 ultramassive dense ETGs of our sample, but, unfortunately, none of these ETGs has an HST optical counterpart.

Given this, we collected a sample of galaxies from literature that is included in the Blanton catalogue with $R_{\mathrm{e}}$ estimated on HST images as well. We firstly refer to the COSMOS catalogue described in Sect. 2.2. In Fig. A.1 the $R_{\mathrm{e}}$ of galaxies at $0.1<z<$ 0.4 measured on HST/ACS-F814W images ( $\sim r$-band rest frame) by Sargent et al. (2007) is reported versus the $R_{\mathrm{e}}$ ( $r$-band) taken from the Blanton release (blue points). The match between the two releases do not include galaxies with $R_{\mathrm{e}}$ smaller than $2 \mathrm{kpc}$, hence, to investigate the reliability of Blanton estimates even at lower effective radii, we integrate the sample with galaxies taken from the ACS-Coma Cluster Survey (Carter et al. 2008). The effective radii of Coma galaxies were measured on the $F 814 \mathrm{~W}$ images (exposure time $\sim 1400 \mathrm{~s}$ ) with GALFIT (Hoyos et al. 2011) and, in Fig. A.1, are compared with the $i$-band SDSS $R_{\mathrm{e}}$. The range of $R_{\mathrm{e}}$ covered by the two samples $\left(1 \mathrm{kpc} \lesssim R_{\mathrm{e}} \lesssim 6 \mathrm{kpc}\right)$ is very similar to that covered by our sample of ultramassive dense local ETGs. The figure shows that, in the range of radius of our ultramassive dense ETGs ( $\left.1 \mathrm{kpc} \lesssim R_{\mathrm{e}} \lesssim 6 \mathrm{kpc}\right)$, the estimates by Blanton agree with those measured for HST images $\left(R_{\mathrm{e}, \mathrm{HTS}}=1.03 \pm 0.08 R_{\mathrm{e}, \mathrm{NY}}+0.06 \pm 0.24\right)$.

\section{A.2. Stellar mass of local SDSS ETGs}

SDSS galaxies are provided with different estimates of stellar masses. In our analysis we decided to adopt the stellar masses derived through the SED fitting for consistency with high and intermediate redshift stellar mass estimates. To show that our conclusions are not affected by our choice of $M_{\star}$, in this Appendix we compare two different estimates of this quantity to asses the consistency of the measures, at least in the range of values we are interested in.

In the left panel of Fig. A.2 we plot the stellar mass derived through the fit of the multiband photometry (used in this paper, $M_{\star \text {,photo }}$ ) versus that obtained fitting the spectral indices ( $M_{\star \text {,index }}$, Kauffmann et al. 2003).

The figure shows that for the bulk of local ETGs with $M_{\star, \text { photo }}>10^{11} M_{\odot}$ the two estimates agree well, but a few

\footnotetext{
5 (http://www2.cadc-ccda.hia-iha.nrc-cnrc.gc.ca/ en/search/?collection=CFHTMEGAPIPE\&noexec=true\# queryFormTab).
}

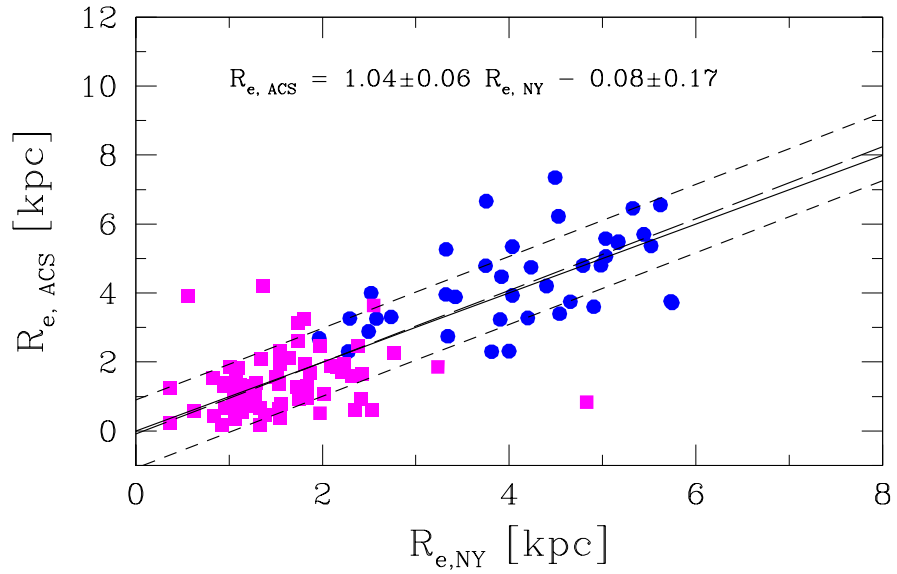

Fig. A.1. Effective radius measured on $F 814 W / H S T$ images for galaxies at $0.1<z<0.4$ (blue points) and for galaxies in Coma cluster (magenta squares) compared with the estimates obtained on SDSS images by Blanton et al. (2005) in $r$-band, for galaxies at $0.1<z<0.4$, and in $i$-band images for Coma galaxies. Solid line is the $1: 1$ correlation, long-dashed line is the best fit relation obtained with a sigma clipping algorithm, and dashed lines indicate the $1 \sigma$ deviation from the best fit.

percent of these significantly deviate from the main correlation. In the figure, we identified these outliers, represented in the figure by squares, as those galaxies at 3 sigma from the best-fit relation derived with a sigma clipping algorithm. We notice that a significant portion of outliers below the relation has $M_{\star \text {,photo }}>10^{11} M_{\odot}$, while $M_{\star, \text { index }}<10^{11} M_{\odot}$. Among these ultramassive outliers, we highlighted with open blue squares those with $\Sigma>2500 M_{\odot} \mathrm{pc}^{-2}$, i.e. those included in the local sample of ultramassive dense local ETGs. To identify the more realistic stellar mass estimate for these ultramassive outliers, in the central panel we plot the velocity dispersion derived from the virial theorem $\left(\sigma_{\text {vir }}\right)$

$\sigma_{\mathrm{vir}}=\sqrt{G M_{\star, \text { photo }} / \beta R_{\mathrm{e}}}$

where $G$ is the gravitational constant and $\beta=(8.87-0.831 \times$ $n+0.0241 \times n^{2}$ ), following Cappellari et al. (2006), versus the measured dispersion $\left(\sigma_{\mathrm{dr} 7}\right)$. For the velocity dispersions of local ETGs, we used the DR7 $\sigma$; see Thomas et al. (2013) for more tests on the reliability of DR7 $\sigma$ estimates.

The majority of ultramassive outliers deviate from the general trend and, in particular, at fixed $\sigma_{\mathrm{dr} 7}, \sigma_{\mathrm{vir}}$ should be lower. This suggests that the $M_{\star \text {,photo }}$ for these galaxies are overestimated. As further confirmation, in the right panel we plot $\sigma_{\mathrm{dr} 7}$ versus $\sigma_{\text {vir }}$ but with the last quantity derived from the $M_{\star \text {,index }}$ estimates. We observe that in this case, the ultramassive outliers follow the main relation.

In fact, most of the ultramassive outliers despite having $M_{\star, \text { photo }}>10^{11} M_{\odot}$, have $M_{\star}<10^{11} M_{\odot}$ and thus we exclude them from our sample of local ultramassive dense ETGs.

\section{Appendix B: Insights into the impact of $\boldsymbol{R}_{e}$ definition on the samples of local dense galaxies}

In Sect. 2.5.1, we have shown that the number of local massive dense galaxies is drastically dependent on the $R_{\mathrm{e}}$ used. In particular, we have shown that in the spectroscopic SDSSDR7 release, the number of galaxies with $M_{\star}>10^{11} M_{\odot}$, $\log \left(R_{\mathrm{e}} / \mathrm{kpc}\right)<0.56 \times\left(\log \left(M_{\star} / M_{\odot}\right)-9.84\right)-0.3$, and $^{0.1}(u-r)>$ 2.5 is $\sim 10$ if the semi-major axis obtained fitting a de Vaucouleur 

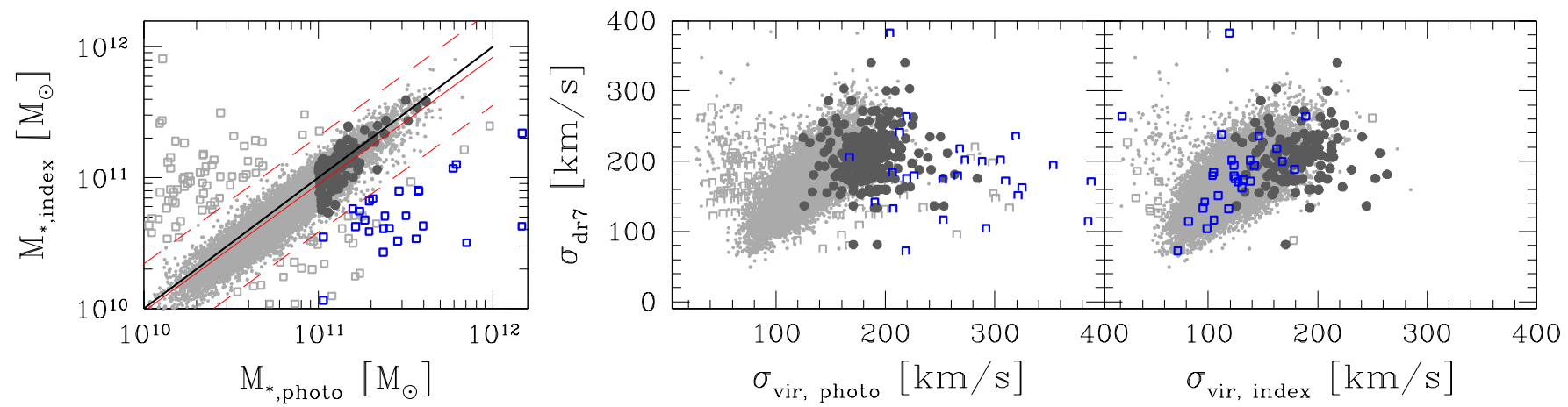

Fig. A.2. Left panel: stellar mass derived through the fit of the multiband photometry (used in this paper, $M_{\star \text {,photo }}$ ) vs. that obtained fitting the spectral indices $\left(M_{\star \text {,index }}\right.$, Kauffmann et al. 2003) for local ETGs at $0.05<z<0.1$ (light grey points). Black solid line is the 1:1 correlation, while red line is the best-fit relation and red-dotted curves are the $3 \sigma$ lines. All of the points at $>3 \sigma$ are indicated as open squares. Among these outliers, blue squares are those with $M_{\star}>10^{11} M_{\odot}$, and $\Sigma>2500 M_{\odot} \mathrm{pc}^{-2}$, i.e. nominally included in local sample of ultramassive dense local ETGs. Dark grey points are the remaining part of the ultramassive dense local ETGs. Central panel: the measured velocity dispersion $\left(\sigma_{\mathrm{dr} 7}\right)$ vs. the velocity dispersion derived from the virial theorem assuming the $M_{\star \text {,photo }}$ values as mass $\left(\sigma_{\text {vir,photo }}\right)$. Right panel: the same of central panel but with $\sigma_{\text {vir }}$ derived assuming the $M_{\star \text {,index }}$ values.
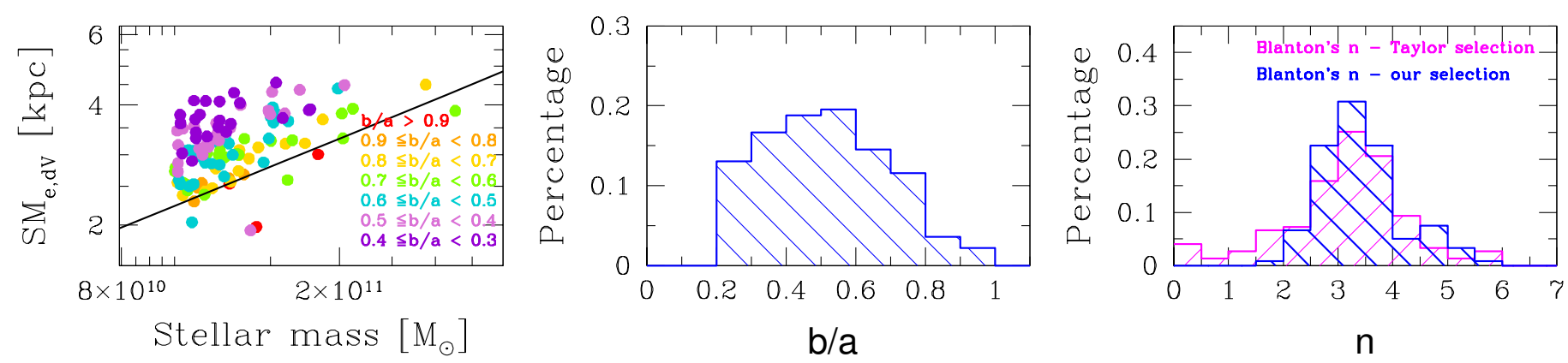

Fig. B.1. Left panel: sample of local dense galaxies selected using the Taylor et al.'s cuts and circularized de Vaucouleur radius $R_{\mathrm{e}, \mathrm{d} V}$, and plotted in the size-mass plane using their semi-major de Vaucouleur axis $\mathrm{SM}_{\mathrm{e}, \mathrm{d} V}$. Galaxies are colour coded according to their $b / a$ ratio, as declared in the labels. Solid line indicates the $\log \left(R_{\mathrm{e}, \mathrm{d} V} / \mathrm{kpc}\right)=0.56 \times\left(\log \left(M_{\star} / M_{\odot}\right)-9.84\right)-0.3$ relation. Central panel: the distribution of the axis ratio $b / a$ for the same galaxies in the left panel. Histogram counts are normalized to unity. Right panel: the distribution of the Sersic index $n$ of local dense galaxies selected using the Taylor et al. cuts and circularized Sersic radius (blue histogram). Histogram counts are normalized to unity. Magenta histogram is the distribution of the Sersic index for our sample of ultramassive dense ETGs.

profile $\left(\mathrm{SM}_{\mathrm{e}, \mathrm{d} V}\right)$ is used and increases up to $\sim 190$ if the circularized de Vaucouleur one $\left(R_{\mathrm{e}, \mathrm{d} V}\right)$ is used instead. Hereafter, for the sake of convenience, we refer to this last sample as the circularized sample. This implies that circularization (hence the axis ratio $b / a$ ) has a huge impact on the selection of the sample. In order to better visualize the impact of the $b / a$ ratio on the selection of the sample, in the left panel of Fig. B.1 we plotted the galaxies of the circularized sample with reliable stellar mass (see Sect. 2.5.1) in the size-mass plane, using for each galaxy its semi-major axis $\mathrm{SM}_{\mathrm{e}, \mathrm{d} V}$. We colour coded the galaxies according to their $\mathrm{b} / \mathrm{a}$ ratio. The solid line indicates the relation $\log \left(R_{\mathrm{e}, \mathrm{d} V} / \mathrm{kpc}\right)=0.56 \times\left(\log \left(M_{\star} / M_{\odot}\right)-9.84\right)-0.3$. As expected, the figure shows that $\sim 10$ galaxies have $\log \left(R_{\mathrm{e}, \mathrm{d} V_{n c}} / \mathrm{kpc}\right)$ $<0.56 \times\left(\log \left(M_{\star} / M_{\odot}\right)-9.84\right)-0.3$, while all of the others lie above the line with a b/a ratio that progressively decreases with the distance from the solid line, down to $b / a \simeq 0.3$. In the central panel of Fig. B.1, we reported the distribution of the axis ratio b/a for the same sample of galaxies. Half of the sample has $b / a<0.5$ and this is the main reason for the drastic difference between the samples selected using circularized $R_{\mathrm{e}}$ and semimajor axis $\mathrm{SM}_{\mathrm{e}}$. The leftmost panel of Fig. B.1 also shows that only the galaxies with $b / a \gtrsim 0.8-0.9$ are included in both samples and this is the main reason why, even if our ultramassive dense ETGs are expected to be mostly roundish systems, i.e. objects for which their semi-major axes should be similar to their $R_{\mathrm{e}}$, Taylor et al. do not selected them in their sample. In fact, we have checked that only 5\% (six galaxies) of ETGs in our sample have $b / a>0.8$, which are exactly those ETGs that satisfy the Taylor conditions.

Finally, in Sect. 2.5.1 we found that the number of local galaxies with $M_{\star}>10^{11} M_{\odot}, \log \left(R_{\mathrm{e}} / \mathrm{kpc}\right)<0.56 \times\left(\log \left(M_{\star} / M_{\odot}\right)\right.$ $-9.84)-0.3,{ }^{0.1}(u-r)>2.5$ still increases if circularized Sersic $R_{\mathrm{e}}$ are used (in particular, only $\sim 45 \%$ of the galaxies are included in the sample selected using circularized de Vaucouleur $R_{\mathrm{e}}$ ). This result, as claimed in Sect. 2.5.1, is expected, because dense galaxies are known to have a Serisc index that is lower than 4. In the right panel of Fig. B.1 we reported the distribution of the Sersic index $n$ for the local dense galaxies selected using the Taylor et al. cut and circularized Sersic $R_{\mathrm{e}}$ : more than $80 \%$ of the galaxies have $n<4$. There is also a tail of galaxies with a Sersic index that is lower than 2 . This is mainly because galaxies are selected with a colour cut, and it is known that there is a non-negligible fraction of red disks. Actually, for comparison, the magenta histogram in the right panel of Fig. B.1 shows the distribution of the Sersic index for our sample of ultramassive dense ETGs. It is nice to observe how the tail at $n<2$ 


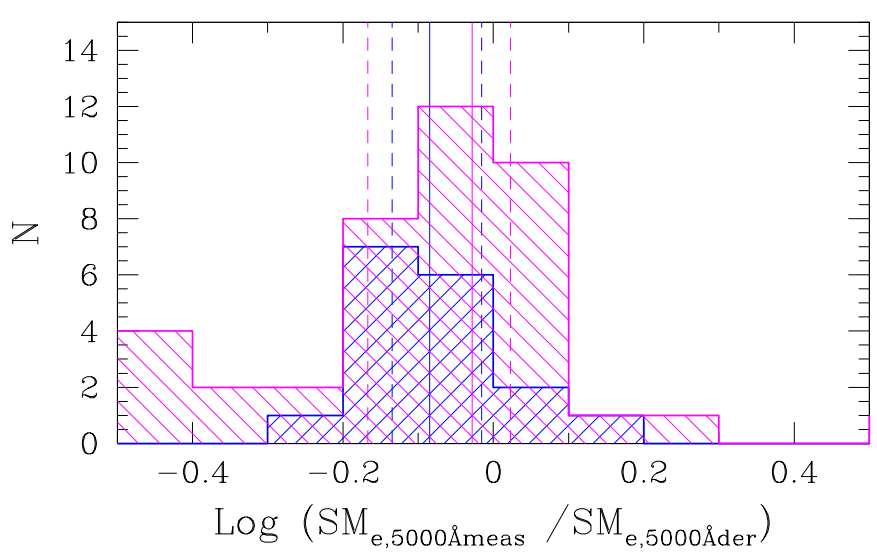

Fig. C.1. Distribution of the $\mathrm{SM}_{\mathrm{e}, 5000} \AA$ meas $/ \mathrm{SM}_{\mathrm{e}, 5000} \AA$ der ratio as function of the redshift. SMR $R_{\mathrm{e}, 5000 \AA \text { meas }}$ is the semi-major axis at $5000 \AA$ rest frame measured directly on the images, while $\mathrm{SM}_{\mathrm{e}, 5000 \text { Ådev }}$ is the same quantity, but inferred from F125W-band images using Eq. (2) from van der Wel et al. (2014). The blue histogram refers to ETGs at $0.5<z<0.7$, while the magenta histogram refers to ETGs at $0.7<z<0.9$. Solid lines are the median values of the distributions and dotted lines are the median value of the distribution at 25 and 75 percentile.

disappears (no selection on the Sersic index is imposed a priori to the local sample of ETGs, or to any other sample used in our analysis), strengthening the robustness of the visual classification in selecting spheroidal galaxies.

\section{Appendix C: The effect of the extrapolation on size measurements}

In Sect. 2.5.3, we have shown that the extrapolation of the size of a galaxy via Eq. (2) by van der Wel et al. (2014) has an impact on the number of dense galaxies, therefore, in this Appendix, we aim to deepen the effect of the extrapolation on size measurements. In Fig. C.1, for the two bins of redshift $0.5<z<0.7$, and $0.7<z<0.9$, we reported the distribution of $\log \left(\mathrm{SM}_{\mathrm{e}, 5000 \AA \AA} \AA_{\text {meas }} / \mathrm{SM}_{\mathrm{e}, 5000} \AA\right.$ der $)$ for a sample of elliptical galaxies, where $\mathrm{SM}_{\mathrm{e}, 5000} \AA$ meas is the semi-major axis at $5000 \AA$ rest frame measured directly on images and $\mathrm{SM}_{\mathrm{e}, 5000} \AA$ der is that derived from $\mathrm{SM}_{\mathrm{e}, F 125 W}$ using Eq. (2) by van der Wel et al. (2014). For the galaxies at $0.5<z<0.7$, we refer to a subsample of COSMOS ellipticals. More specifically, for the $\mathrm{SM}_{\mathrm{e}, 5000} \AA$ meas values we refer to the measurements by Sargent et al. (2007) on the $F 814 \mathrm{~W}$-band images and discussed in Sect. 2.2, while for the $\mathrm{SM}_{\mathrm{e}, F 125 W}$ values we considered the estimates by van der Wel et al. (2012). We crossmatched the two catalogues and selected only elliptical (ZESTtype $=1$ ) galaxies with $M_{\star}>10^{10} M_{\odot}$. For the galaxies at $0.7<z<0.9$, we considered a sample of elliptical galaxies

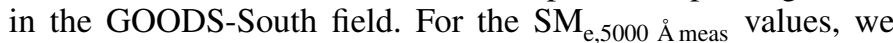
used our sample of ETGs on GOODS-South (Tamburri et al. 2014) with the $\mathrm{SM}_{\mathrm{e}}$ derived from F850LP images (briefly described in Sect. 2.1), and for the $\mathrm{SM}_{\mathrm{e}, F 125 \mathrm{~W}}$ measurements we considered the catalogue by van der Wel et al. (2012). At $0.5<$ $z<0.7, \mathrm{SM}_{\mathrm{e}, 5000} \AA$ der overestimates the $\mathrm{SM}_{\mathrm{e}, 5000} \AA$ meas by a factor -0.08 dex (median value) and, in particular, for more than $80 \%$ of the galaxies $\mathrm{SM}_{\mathrm{e}, 5000 \AA \text { meas }} / \mathrm{SM}_{\mathrm{e}, 5000 \AA \text { der }}<1$. At $0.7<z<0.9$, where the correction is extrapolated over a shorter wavelength range, this fraction decreases to $\sim 65 \%$ and the median value is -0.02 . The two samples of galaxies at $0.5<z<0.7$ and $0.7<z<0.9$ do not have exactly the same distribution in stellar mass and this can alter the relative comparison of

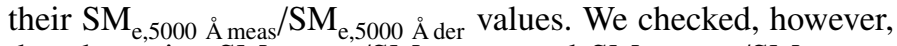
that the ratios $\mathrm{SM}_{\mathrm{e}, F 814 W} / \mathrm{SM}_{\mathrm{e}, F 125 W}$ and $\mathrm{SM}_{\mathrm{e}, F 850 L P} / \mathrm{SM}_{\mathrm{e}, F 125 W}$ do not depend on stellar mass, as stated by van der Wel et al. (2014). Although a test on more statistically significant samples would be necessary to be conclusive, Fig. C.1 shows that an average variation of $\mathrm{SM}_{\mathrm{e}}$ with $\lambda$ (as assumed in the Eq. (2) by van der Wel) could not account for the variety of stellar populations in elliptical galaxies at high redshift (see e.g. Guo et al. 2011; Gargiulo et al. 2012), and this should be kept in mind in the analysis, interpretation and comparison of the results.

\section{Appendix D: Velocity dispersion measurements at $z \sim 1.4$}

Figure 3 shows the FORS2 spectra of the five galaxies of our high- $z$ sample. As expected from the redshift estimates based on the continuum shape provided by AMICI spectra in the framework of the MUNICS survey, for the four galaxies at $z \lesssim 1.4$ (ID $511,633,527,389)$ we detected many absorption features (e.g. CN(3833 $\AA$ ), the CaII K and H (3933+3968 $⿱$ A), blue lines) in addition to the $4000 \AA$ break. We notice that the ETG 527, whose elliptical morphology is confirmed by the HST image and surface brightness profile (see Fig. 1), clearly shows the $\mathrm{O}_{2}$ emission line at $3727 \AA$. We exclude that the presence of this emission line is due to AGN activity. In facts, the five ETGs of our sample were observed with XMM observations and only the source 443 has an X-ray detection (Severgnini et al. 2005). For galaxy 443, we detected the MgII absorption feature, as expected, however, we notice that the detection of this absorption line fixes the redshift of this galaxy at $z=1.91$ which is slightly higher than the value originally estimated from the AMICI continuum $(z=1.70 \pm 0.05)$. In Table 5 we report the values of the redshifts for the five galaxies of our sample.

The tool pPXF we adopted to derive the velocity dispersion describes the line-of-sight velocity distribution (LOSVD) as a Gauss+Hermite polynomial to take possible deviation of the line profile from a pure Gaussian shape into account. Following the prescription of the pPXF documentation, we ran simulations to define the value of the BIAS parameter, which is a penalty term that biases the solution towards a Gaussian shape if the higher moments of Hermite polynomial are not constrained from the data. We found that for our galaxies this term ranges between 0.1 and 0.2, which is in agreement with Cappellari et al. (2011).

Once we fixed the BIAS level, we measured the velocity dispersion by means of a weighted fit and adopted a set of synthetic stars as templates (see Sect. 3.1). To check for possible dependence of the $\sigma$ estimate on the selected templates, we repeated the measure adopting a set of BC03 models with solar metallicity, $\tau=0.1 \mathrm{Gyr}$, and varying age. We found that the $\sigma$ values are perfectly comparable (differences $<5 \%$ ) with those obtained with the Munari stars. For clarity, the values reported in this paper and used in the analysis refer to $\sigma$ estimates derived adopting the set of stars as templates.

We included in the fitting function both additive and multiplicative polynomials to take into account a possible residual template mismatch and eventual sky subtraction and spectral calibration errors. We verified the stability of our measures varying the degree (up to 6) of the two sets of polynomials and found that the detected variations in the estimates of velocity dispersion are lower than $3 \%$, which is well within $1 \sigma$ error bar.

Four out of five ETGs of our sample have spectra covering the rest-frame wavelength range $\sim[0.3-0.43] \mu \mathrm{m}$, thus 


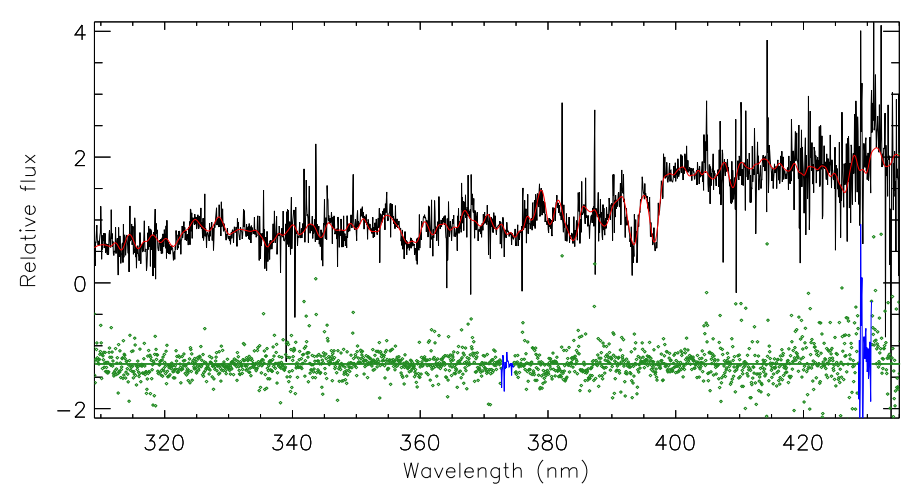

Fig. D.1. Example of the galaxy spectrum template (black line) we constructed for the real galaxy S2F1-633. The red line denotes the best-fit model, while the green points show the residuals.

including the $4000 \AA$ break and many absorption features in addition to $\mathrm{Ca} \mathrm{H}+\mathrm{K}$. In the past, several analyses have reported that the inclusion of the $\mathrm{H}+\mathrm{K}$ features leads to an overestimate of the $\sigma$ value, up to a factor of $\sim 20 \%$ (Kormendy 1982; Kormendy \& Illingworth 1982). These lines are intrinsically broad $(\sim 5 \AA)$ and, in addition, the $\mathrm{CaH}$ line at $3968 \AA$ is blended with $\mathrm{H} \epsilon$ at $3969 \AA$. Moreover, error in fitting the steep continuum gradient at $4000 \AA$ could produce a template mismatch. All of these factors can invalidate the measured dispersions. Nonetheless, the real impact of these feature on the $\sigma$ estimates is not quantifiable a priori (e.g. Treu et al. 2001; van de Sande et al. 2013), thus we organized a set of simulations to test the reliability of our velocity dispersion measurements.

Briefly, for each galaxy of our sample, we created four model galaxy spectra starting from a simple stellar population model from Maraston \& Strömbäck (2011) with solar metallicity and ages 2, 3, 4, 5 Gyr. We redshifted the spectra to the galaxy redshift, degraded them to the dispersion of real spectrum ( $\sim 55 \mathrm{~km} \mathrm{~s}^{-1}$ per pixel), and added to them, pixel-by-pixel, a fake noise randomly extracted by the real noise. These template spectra were convoluted with a Gauss+Hermite LOSVD with the $h_{3}$ and $h_{4}$ Hermite moment equal to those measured on the real spectrum and velocity dispersion $\left(\sigma_{\text {in }}\right)$ varying in the range $\sim$ [200-500] $\mathrm{km} \mathrm{s}^{-1}$ over 500 steps. We fixed the line-of sight velocity $V$ to zero. As an example, in Fig. D. 1 we report a galaxy spectrum template that we constructed for the galaxy S2F1-633 with velocity dispersion $400 \mathrm{~km} \mathrm{~s}^{-1}$ (black line). With the same star templates used on real spectra, we measured their velocity dispersions $\left(\sigma_{\text {out }}\right)$, assuming a Gauss + Hermite polynomial as a fitting function. In Figs. D.2-D.5, and D.6 we present the results of the simulations for the galaxy S2F1-511, S2F1-633, S2F1527, S2F1-389, S2F1-443.

The results we obtained show that, irrespective of the stellar population properties, for all of the galaxies of our sample, fitting a Gauss+Hermite polynomial over the whole wavelength range covered by our spectra, with a penalty BIAS $\simeq 0.1$, is the best choice to recover the intrinsic value of velocity dispersions. The same set of simulations for galaxy 443 shows the feasibility of extracting a reliable estimate of velocity dispersion from spectrum covering the blue wavelength range shortwards the $4000 \AA$ in agreement with that found by Cappellari et al. (2009).

In fact, the difference, $\sigma_{\text {out }}-\sigma_{\text {in }}$, shows a slight offset that is always lower than the dispersion of the real spectrum $\left(\sim 55 \mathrm{~km} \mathrm{~s}^{-1}\right.$ per pixel). The exact magnitude of the offset depends on the age and metallicity of the stellar population. We verified that the amount of the offset is not reduced excluding the region around the $\mathrm{Ca} \mathrm{H}+\mathrm{K}$ features from the fit, as done by Kormendy \& Illingworth (1982); for this reason, we decided to include this part of the spectrum in our fit.

Moreover, our simulations have shown that for the $\sigma$ we expect for our galaxies, the median value of the offset between $\sigma_{\text {in }}$ and $\sigma_{\text {out }}\left(\sigma_{\text {off,median }}=\sigma_{\text {out }}-\sigma_{\text {in }}\right)$ depends on the age and metallicity of the input template, but is always smaller than the statistical error on measured velocity dispersion. In Table 5 we reported, for each galaxy, the minimum and maximum value of $\sigma_{\text {off,median }}$ we found in the six sets of simulations when $\sigma_{\text {out }}$ is equal to the value we measured on real spectrum. The Table shows that the offset is never greater than $30 \mathrm{~km} \mathrm{~s}^{-1}$ and can be both positive and negative. Given these two aspects, and our uncertainty on stellar population properties, mostly on metallicity, we decided to not correct our measures for this offset, since we are not able to properly quantify its magnitude. Nonetheless, we note that our results would not change if we corrected the velocity dispersion of each galaxy for the maximum value of its offset.

The errors on velocity dispersion reported in Table 1 are the 25 th and 75th percentile of the distributions of $\sigma_{\text {off }}$ estimated at $\sigma_{\text {out }}$ equal to the velocity dispersion measured on real spectrum. Among the different set of simulations, the quoted errors are extracted from the simulation performed starting from a simple stellar population model with solar metallicity and age equal to that obtained from the fit of the observed SED. However, although the value of the offset is slightly dependent on the stellar population properties, its scatter not. 
A\&A 592, A132 (2016)
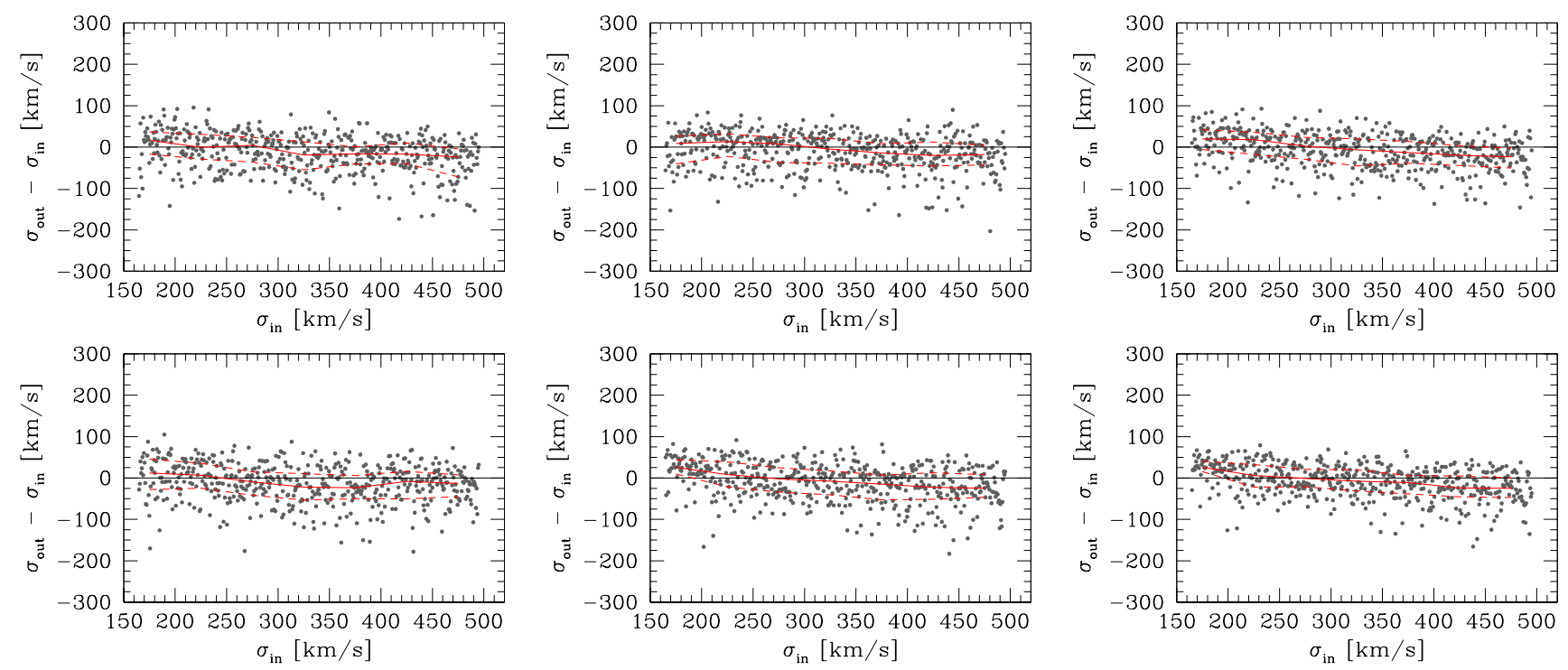

Fig. D.2. Top row: difference between the velocity dispersion measured $\left(\sigma_{\text {out }}\right)$ and that provided as input $\left(\sigma_{\text {in }}\right)$ in the model as function of $\sigma_{\text {in }}$ for galaxy S2F1-511. The template adopted in the simulation have solar metallicity and age 2, 4, 5 Gyr from left to right. It is evident that the age of stellar populations do not affect our measurements. Bottom row: same as top row for the template with age 3 Gyr and subsolar, solar and super-solar metallicity from left to right. The three panels show that our uncertainty on stellar metallicity does not compromise our $\sigma$ estimates. The red solid line is the median of the distribution, while the dashed lines are the 25 th and 75 th percentile.
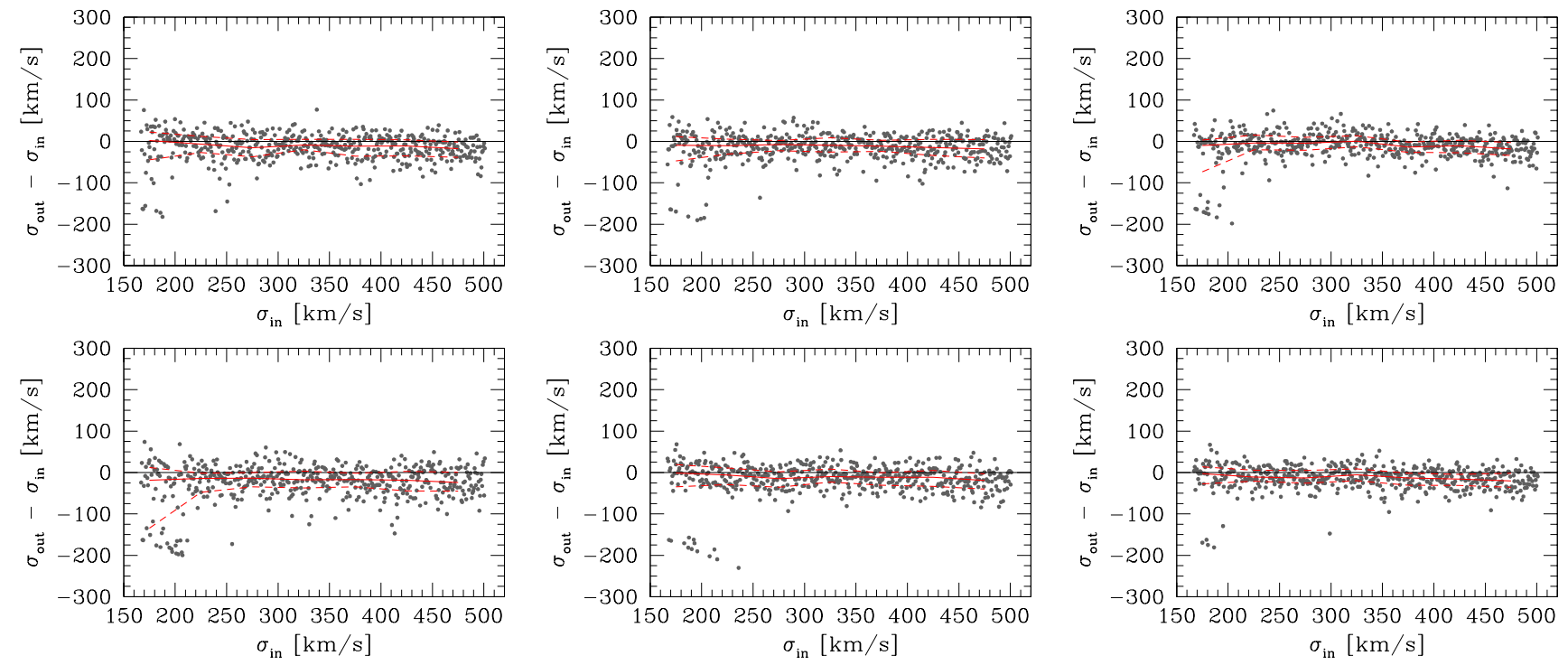

Fig. D.3. Same as Fig. D.2 for galaxy S2F1-633. 
A. Gargiulo et al.: Number density evolution of ultra-massive dense early-type galaxies since $z=1.6$
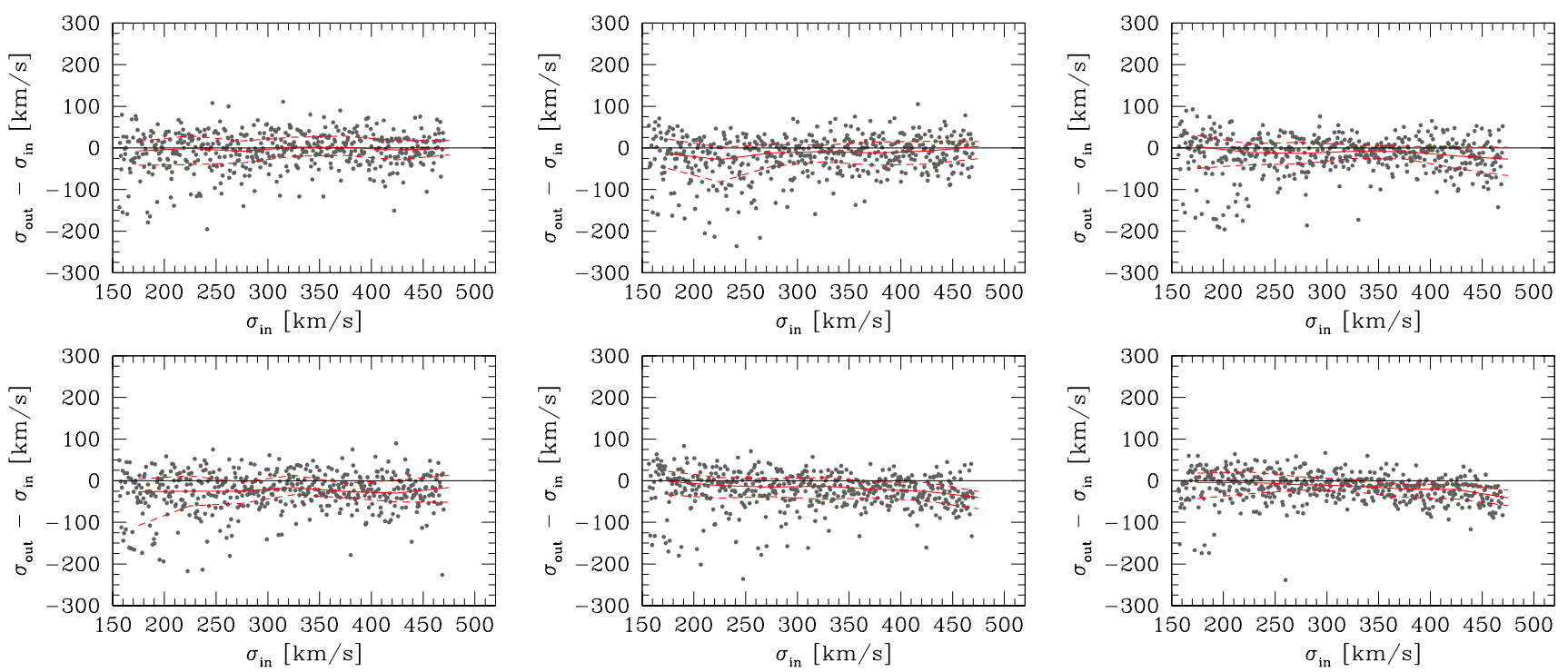

Fig. D.4. Same as Fig. D.2 for galaxy S2F1-527. Given the spectrum and SED fitting of this ETG, which both suggest a very young age for its stellar population, in the simulation of this galaxy we adopted, as galaxy templates, simple stellar population templates with solar metallicity and ages of 0.5, 1, $2 \mathrm{Gyr}$ (upper panels, from left to right) and stellar population templates with an age of 3 Gyr and subsolar, solar, and super-solar metallicity (lower panels, from left to right).
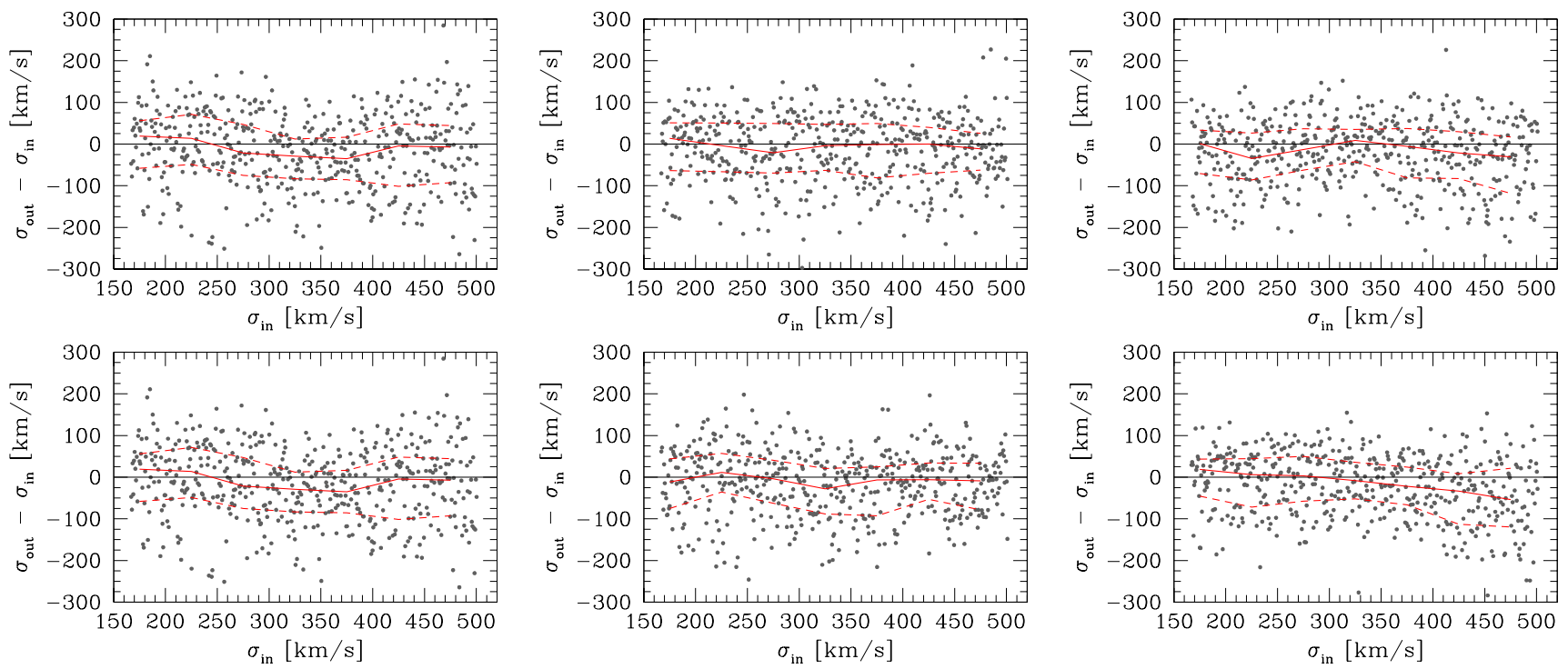

Fig. D.5. Same as Fig. D.2 for galaxy S2F1-389. 
A\&A 592, A132 (2016)
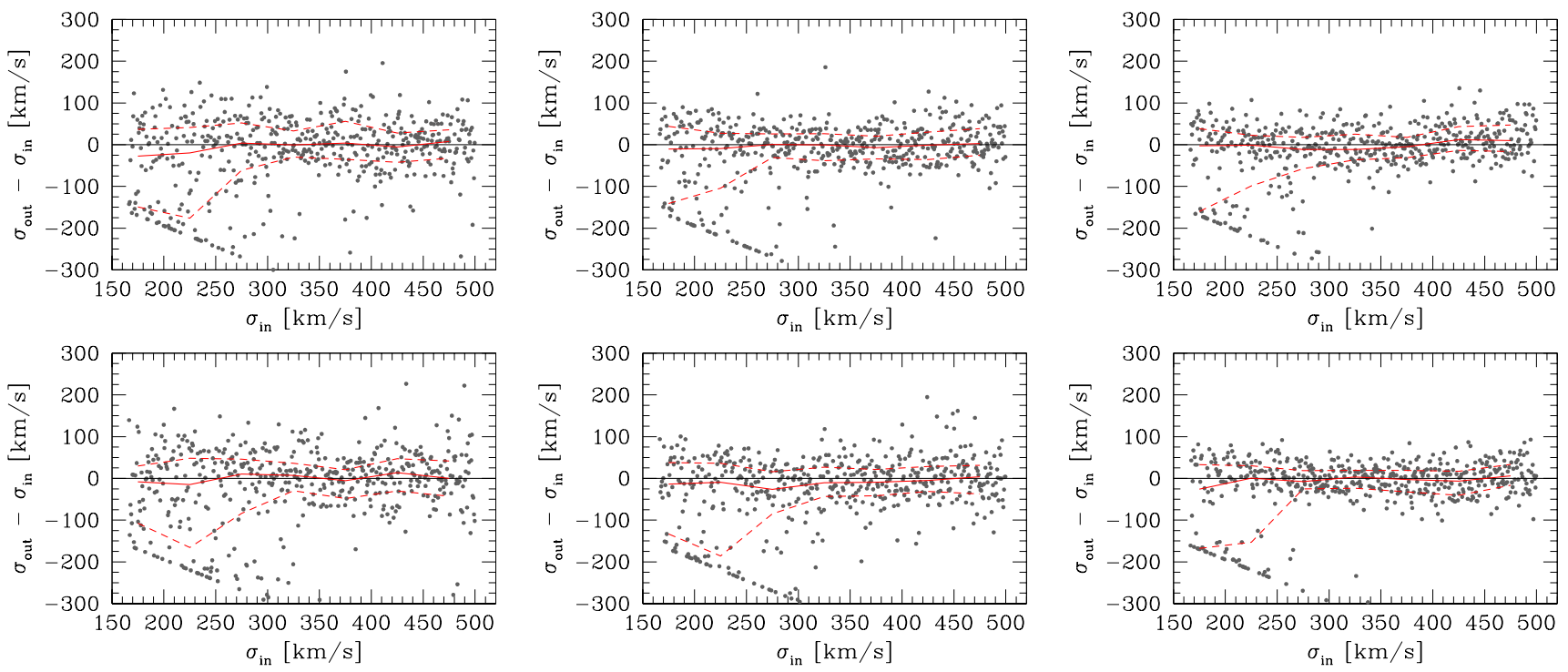

Fig. D.6. Same as Fig. D.2 for galaxy S2F1-443. 